\title{
URBANIZANDO CON TIZA
}

FIG 1

Población total y urbana en Chile entre 1865 y 1970/Country and urban population in Chile between 1865 and 1970 . Gráfico del autor/Author's diagram. Fuente de la información/ Information source: Geisse, 1983, p. 185.

FIG 2

Conventillo/Tenement, c. 1920.

Fuente: Montoya, J. Luces de modernidad: archivo fotográfico Chilectra. Enersis, Santiago, 2001/Source: Montoya, J. Luces de modernidad: Chilectra photographic archive. Enersis, Santiago, 2001.

\section{FIG 3}

Población callampa en el Zanjón de la Aguada, c. 1954/Shanty town in the Zanjón de la Aguada, c. 1954. Fotografía/ Photography: Domingo Ulloa. Fuente/ Source: Biblioteca Nacional de Chile.

En 1930 la agricultura daba trabajo al $\mathbf{1}$ $37,5 \%$ de los chilenos. En 1970, luego de la industrialización del área, esta cifra se redujo al $25 \%$ de la población. Por su parte, los campamentos salitreros del norte habían atraído hacia 1920 a más de 65 mil personas, quienes luego de la quiebra de las mineras migraron en su mayoría al centro del país (Geisse, 1983).
OPERACIÓN SITIO Y VILLA LA REINA [1965-1970]

ntre 1940 y 1960 la ciudad de Santiago duplicó su init población produciendo una crisis habitacional en La capital. Si bien el presidente Eduardo Frei Montalva (1964-1970) planteó el desafío de terminar con la escasez de vivienda, su gobierno no tendría ni el dinero ni el tiempo suficiente para lograr su objetivo a través de las políticas públicas tradicionales. Lo que implementaron fue llamado por sus opositores "Operación Tiza": a mediados de los años sesenta, políticos y planificadores decidieron no construir casas para la gente viviendo en extrema pobreza, sino que hacerlos dueños de un terreno trazado con tiza. Dada la urgencia y falta de dinero, optaron por proveer solo de aquello que la gente no podría obtener por su propia cuenta: diseño urbano, acceso a servicios y equipamientos básicos y la conexión con las redes de la ciudad. A pesar de las dificultades -y no sin errores-en un periodo de cinco años la "Operación Sitio" -nombre formal de esta política pública- entregó cerca de 71 mil sitios a más de 380 mil personas, urbanizando extensas áreas de la periferia del Santiago de la segunda mitad de la década de 1960.

Villa La Reina, en tanto, es un proyecto de autoconstrucción desarrollado en esta época. A diferencia de la "Operación Sitio", este conjunto se desarrolló a escala local, liderado por el arquitecto Fernando

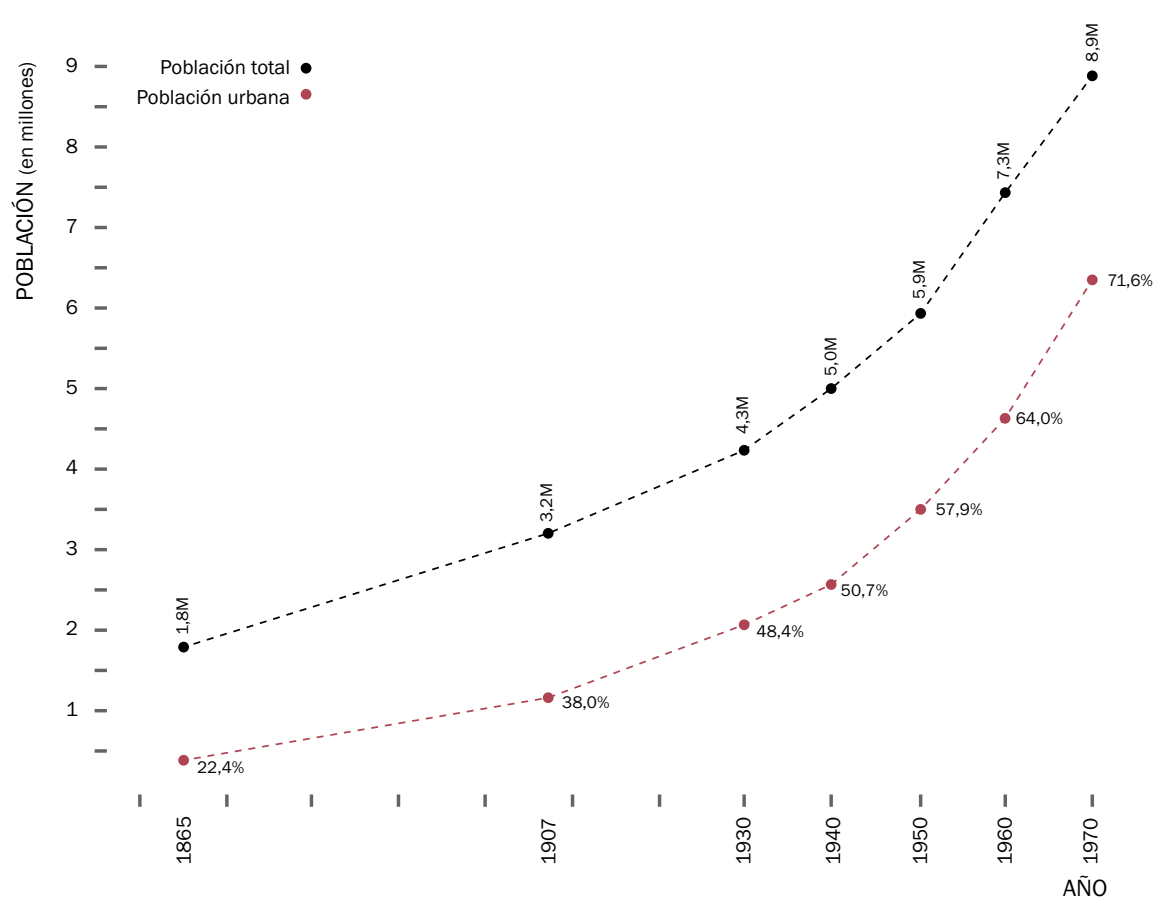

FIG 1

Castillo Velasco, alcalde de la comuna de La Reina entre 1964 y 1968. El trabajo colaborativo entre la alcaldía, la comunidad local y la Universidad Católica, fue uno de los aspectos claves de este proyecto. La organización de los pobladores no solo fue relevante en la autoconstrucción de sus viviendas, sino también en el proceso de diseño y en la creación de un barrio integral con espacio público adecuado, provisto de equipamiento educacional, cercano a nuevas fuentes de empleo y conectado con la estructura de la ciudad.

Si bien la "Operación Sitio" y la autoconstrucción parecían estrategias viables para reducir los costos y acelerar los procesos en vivienda, fueron severamente criticadas. En términos de localización se preveía a principios de la década de los setenta que la "Operación Sitio" derivaría en una masiva segregación social. En términos del proceso de autoconstrucción, el gobierno de Salvador Allende optaría por eliminar este tipo de programas, estableciendo que era un proceso ineficiente y que discriminaba a la población de más escasos recursos.

\section{PRIMERA MITAD DEL SIGLO XX: \\ MIGRACIONES Y ESCASEZ DE VIVIENDA}

Grandes migraciones hacia las ciudades caracterizaron la primera mitad del siglo xx en Chile. Si en 1907 solo un $38 \%$ de las personas vivían en ciudades (1,2 millones de hab.), hacia 1970 este porcentaje se había incrementado a un $71,6 \%(6,4$ millones de hab.) (Geisse, 1983). Esto se debió en gran medida a las migraciones provenientes del campo y de los campamentos mineros del salitre, que habían entrado en bancarrota ${ }^{1}$. Santiago, capital financiera y administrativa del país, atrajo la mayoría de estas migraciones. El resultado fue una enorme escasez de viviendas para mediados de la década del sesenta.

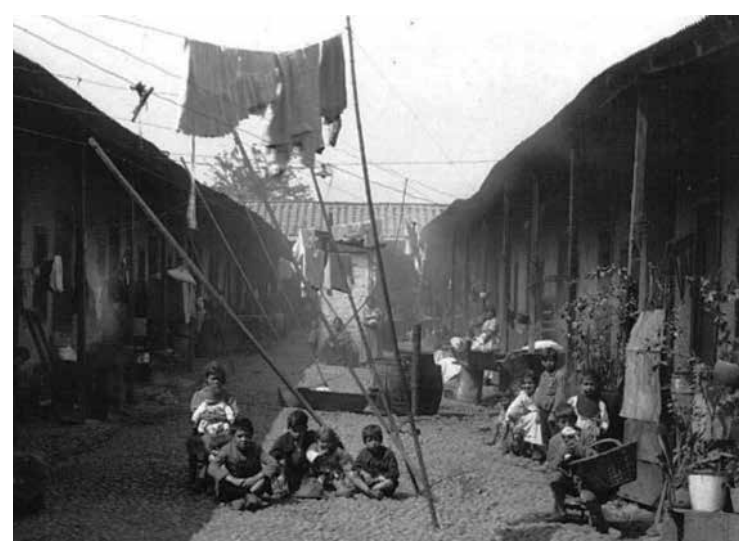

FIG 2 
La Operación Sitio impulsada por Frei Montalva tiene en la Villa La Reina de Fernando Castillo Velasco un caso ejemplar. Ante la escasez, se entregó a los pobladores sólo lo que ellos no podrían obtener por sí mismos: diseño urbano y conexiones con la ciudad.

PALABRAS CLAVE: URBANISMO - CHILE, VIVIENDA SOCIAL, AUTOCONSTRUCCIÓN, DISEÑO URBANO, POLÍTICA DE VIVIENDA.
Frei Montalva promoted the "Operacion Sitio" program and Villa La Reina by Fernando Castillo Velasco is an exemplary case of it. Given scarcity, the people got only what they could not obtain by themselves: urban design and connections with the city.

KEYWORDS: URBANISM - CHILE, SOCIAL HOUSING, SELF-BUILD, URBAN DESIGN, HOUSING POLICIES.

English text in p. 41

\section{FIG 4}

Conjunto Salar del Carmen, Antofagasta/ Salar del Carmen housing, Antofagasta. Arquitectos/Architects Mario Pérez de Arce Lavín y Jaime Besa.

Fuente/Source: CORVI, 1963, p. 21.

\section{FIG 5}

Unidad Vecinal Portales, Santiago. Arquitectos/Architects Bresciani, Valdés, Castillo, Huidobro.

Fotografía/ Photography: René Combeau. Fuente: Archivo Sergio Larraín García Moreno, Pontificia Universidad Católica de Chile/Source: Sergio Larrain García Moreno Archive, Pontificia Universidad Católica de Chile.

\section{FIG 6}

Operación San Gregorio, c. 1959. Cerca de 25.000 ex-habitantes de poblaciones callampas de Santiago cooperaron en la construcción de sus viviendas. Se utilizaron sistemas constructivos a base de paneles prefabricados en madera para acelerar el montaje/Operation San Gregorio, c. 1959. Close to 25,000 exresidents of "poblaciones callampas" from Santiago cooperate in the construction of their homes. They used a pre-fabricated wood panel system to accelerate construction.

Fuente/Source: CORVI, 1963, p. 99
La ciudad de Santiago, desde principios del siglo $\mathrm{xx}$, fue gradualmente transformada en el mayor centro industrial del país. En 1916 el $45 \%$ de la inversión pública en infraestructura se concentró en la capital, ciudad en la que vivía solo el $18 \%$ de la población del país (Geisse, 1983) (fig. 1). La consecuente generación de empleo hacía de Santiago un atractivo destino para las migraciones. En 1907 la ciudad tenía una población de 300 mil habitantes. En 1940 esta cifra se había incrementado a 950 mil y hacia 1960 el número se había duplicado a 1,9 millones de personas, concentrando en Santiago al $58 \%$ de la población urbana del país y produciendo una crisis en la provisión de vivienda. Los pobladores de escasos recursos que llegaban a la capital buscando oportunidades de trabajo y educación no tenían más alternativa que vivir en conventillos o en las llamadas "poblaciones callampa".

Los conventillos eran viviendas precarias desarrolladas por empresarios, que se arrendaban a la gente de menores ingresos. Consistían en dos hileras de habitaciones que compartían un pequeño callejón. La falta de ventilación, de agua potable y alcantarillado era común en estos conjuntos y el hacinamiento era parte de la vida diaria de estas personas. En 1952 Chile tenía más de 160 mil personas viviendo en cuarenta mil de estas habitaciones (CORVI, 1962) (fig 2). Por su parte, las poblaciones callampa eran asentamientos informales ilegales, establecidos en tierras públicas o privadas, generalmente en espacios degradados de la ciudad. En 1952 cerca de 35 mil viviendas formaban diferentes tomas de terreno en las ciudades del país, la mayoría de ellas en Santiago (CORVI, 1962). El objetivo principal de estas poblaciones era vivir cerca de las oportunidades de trabajo y educación que ofrecían las ciudades (fig. 3 ).

En 1953 se fundó la Corporación de la Vivienda

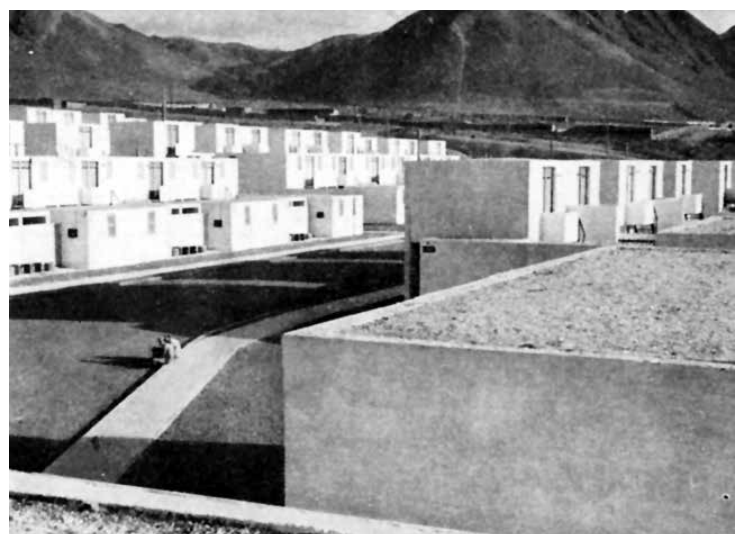

FIG 4

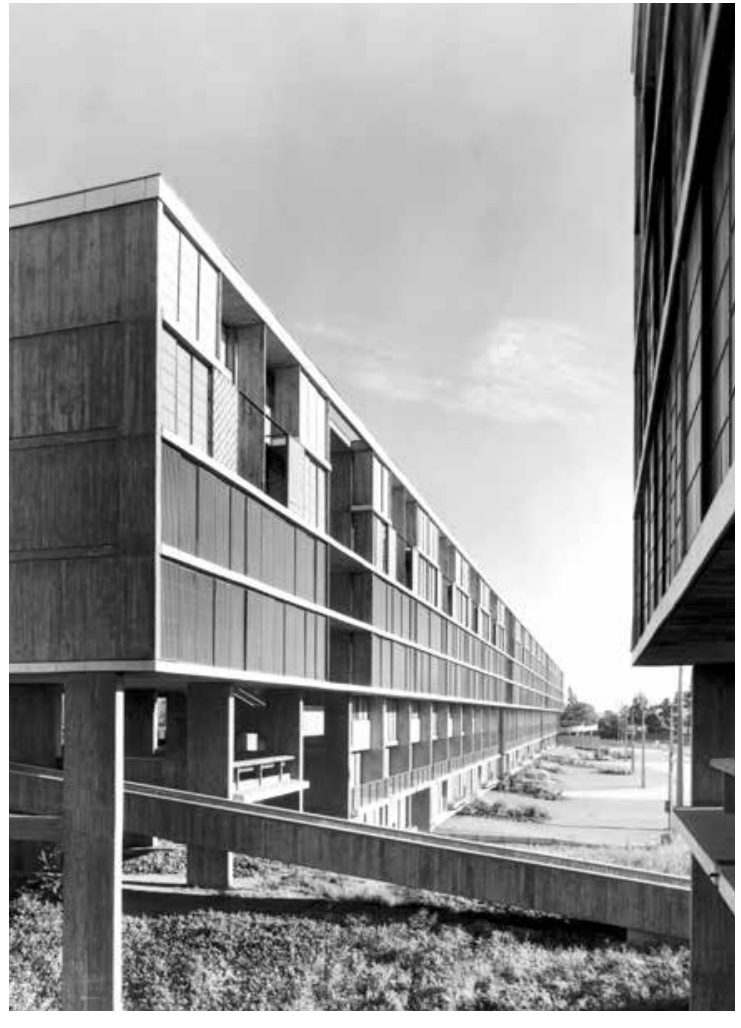

FIG 5

(CORVI) para proveer de soluciones habitacionales al país; en ese año el déficit de viviendas en Chile llegaría a más de 145 mil unidades (CORVI, 1963). La institución abordaría las necesidades tanto de la clase media como de los pobladores de escasos recursos y los proyectos promovidos por esta entidad incluirían desde conjuntos de casas, como la población Salar del Carmen de 1960 -diseñada por Mario Pérez de Arce Lavín y Jaime Besa (fig. 4)- hasta conjuntos de torres y bloques, como la Unidad Vecinal Portales, proyectada en 1958 por la oficina de Bresciani, Valdés, Castillo y Huidobro (fig. 5).

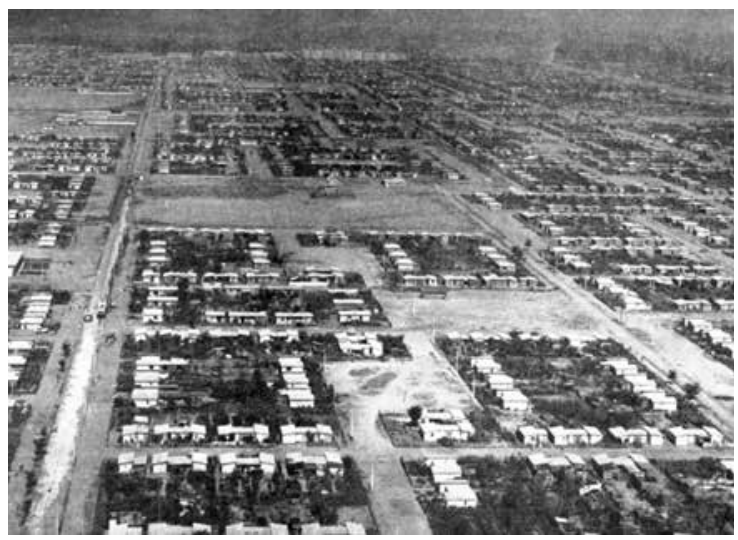

FIG 6 
FIG 7

Ubicación de las Operaciones Sitio construidas entre 1965 y 1970 sobre el plano de Santiago de 2010/Locations of Site Operations built between 1965 and 1970 throughout a plan of Santiago from 2010.

Gráfico del autor/Author's diagram.

Fuente de la información/Information Source: Hidalgo, 2005, p. 293.

FIG 8

Planta Operación Sitio: Población

Conchalí, 1965-66/Operation Site Plan: Población Conchalí, 1965-66.

E./ Sc. 1: 10.000

Fuente/Source: Haramoto, 1985

p. $84-85$.

\section{FIG 9 Y FIG 10}

Operación Sitio: Población Conchalí, 1965-66/Operation Site: Población Conchalí, 1965-66.

450 viviendas fueron construidas por 2.000 personas junto a empresas privadas que proveían sistemas prefabricados de placas y pilares de hormigón para algunas casas, y pino y yeso-cartón para otras/450 homes were built for 2,000 people together with private companies that provided prefabricated panel systems and concrete pillars for some houses, and pine and plasterboard for others.

Fuente fotografías e información/ Photography and information sources: Haramoto, 1985 , p. $84-85$.

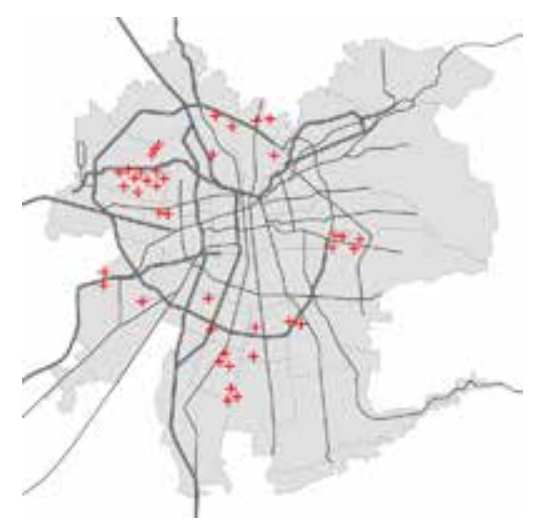

(1)

FIG 7

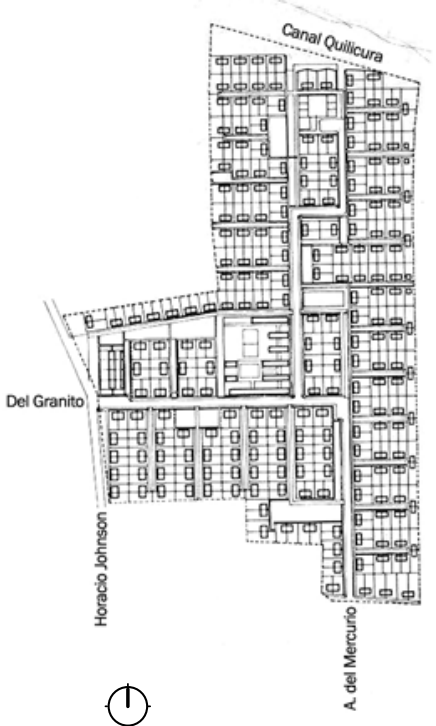

FIG 8
A mediados de los años cincuenta la CORVI comenzó a aplicar estrategias que serían la precursoras de la “Operación Sitio". Los programas de Autoconstrucción y Erradicación consistían en la eliminación de las poblaciones callampa, motivada por el ambiente insalubre en que se encontraban viviendo sus habitantes. CORVI adquiría sitios necesarios para erradicar a los pobladores y les otorgaba el título de propiedad de un nuevo terreno. El lote contenía una pequeña unidad sanitaria de baño y cocina conectada a los servicios básicos. Las viviendas definitivas serían construidas en gran medida por los propios pobladores (CORVI, 1963) (fig. $6)$.

La autoconstrucción surgió informalmente en diversos países latinoamericanos durante los cincuenta y sesenta. Estas poblaciones fueron llamadas "barriadas" en Perú, "villas miseria" en Argentina, "favelas" en Brasil, y "poblaciones callampa" en Chile². El arquitecto John Turner estudió en terreno el proceso de autoconstrucción y lo promovió internacionalmente como una solución factible ante el problema habitacional de las personas viviendo en extrema pobreza. De acuerdo a sus observaciones en Lima, la estrategia tras la autoconstrucción era más apropiada que las que soluciones otorgadas por gobiernos o empresas privadas. En primer lugar, se trataba de una solución más económica que la provista por la vía tradicional. Los pobladores proveían la fuerza laboral para la construcción de casas que respondían de forma más precisa a sus necesidades, y no a viviendas tipo. Finalmente, durante el proceso se generaban las primeras redes sociales necesarias para el futuro desarrollo de los conjuntos. Turner argumentaba de todas formas que instituciones públicas debían apoyar estas construcciones a través de apoyo técnico, urbanización de los sitios, provisión de terrenos y materiales para la construcción (Turner, 1976; Turner y Fichter, 1972).

\section{EL DERECHO A LA VIVIENDA EN EL GOBIERNO DE EDUARDO FREI MONTALVA}

Durante el gobierno de Eduardo Frei Montalva la vivienda se definió como "un bien de primera necesidad al que tiene derecho toda familia... cualquiera sea su nivel socio-económico" (Haramoto, 1980, p. 29). Siguiendo este planteamiento, el Estado ayudaría económicamente a las familias

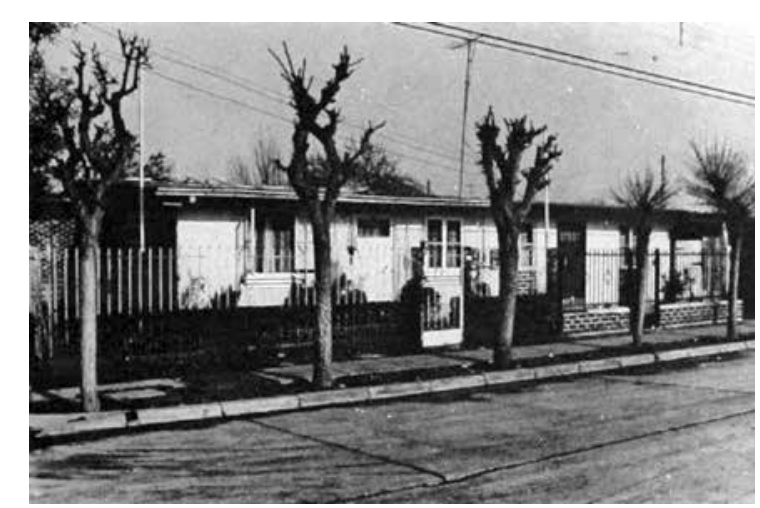

FIG 10
En Río de Janeiro el $20 \%$ de la población 2 vive en favelas actualmente, mientras que las áreas urbanas informales en Caracas y Lima alcanzan el 60\% y $70 \%$ respectivamente (Jáuregui, 2009).

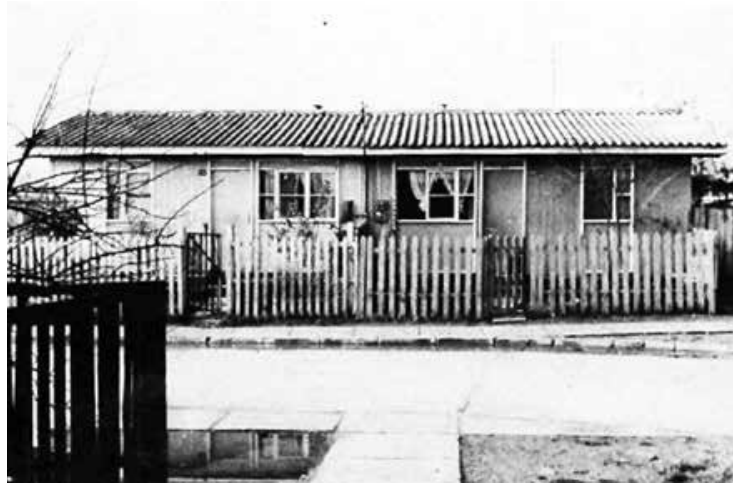

FIG 9 


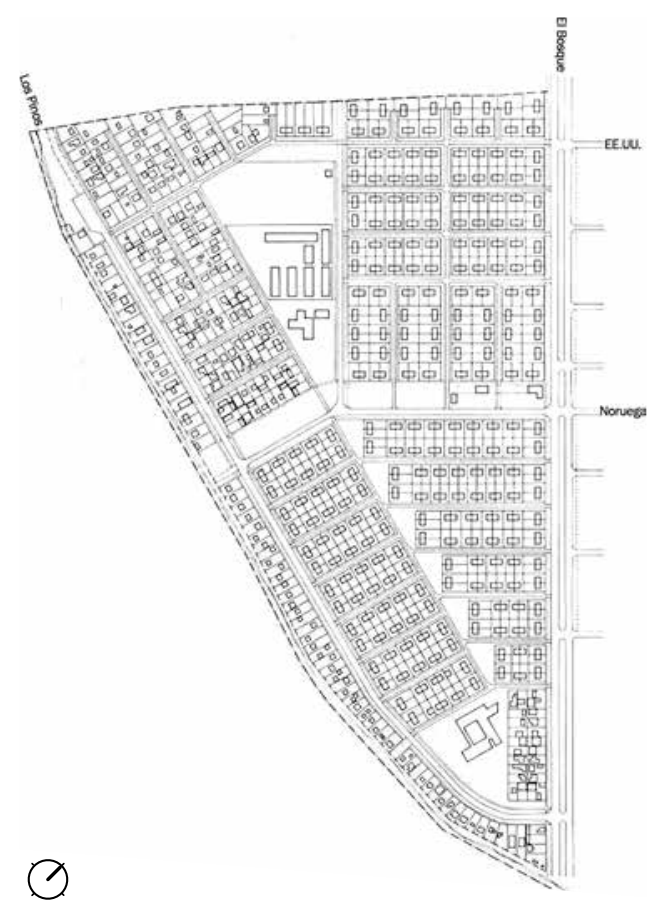

FIG 11

Planta Operación Sitio: Población El Bosque, 1970/Operation Site plan:

Población El Bosque, 1970.

E./Sc. 1: 10.000 .

Fuente/Source: Haramoto, 1985,

p. $88-89$

\section{FIG 12 Y FIG 13}

Operación Sitio/Operation Site: Población El Bosque, 1970.

744 viviendas para 2.976 personas $/ 744$ homes for 2,976 people.

Fuente fotografías e información/ Photography and information sources: Haramoto, 1985 , p. 88-89. de escasos recursos que no pudieran acceder a una vivienda. En el caso de las personas viviendo en extrema pobreza, el Estado no financiaría el total de la vivienda, sino que haría parte a los pobladores de un proceso de autoconstrucción de sus propios conjuntos habitacionales. Se buscaba integrar a esta población a las redes de la ciudad, evitando que continuaran viviendo en asentamientos informales, ineficientes en su distribución espacial e insalubres dada la precariedad de las viviendas y a la ausencia de servicios como agua potable y alcantarillado. Bajo esta lógica sería promovida la "Operación Sitio".

En 1965 se creó el Ministerio de Vivienda y Urbanismo (MINVU), responsable, entre otras funciones, de elaborar los planes de vivienda y desarrollo urbano. Los programas elaborados en este tiempo no pretendían reducir la crisis habitacional a un problema de escasez de viviendas, sino que tuvieron por objetivo la edificación de conjuntos integrales; de esta forma se planificó la construcción de los equipamientos necesarios para el desarrollo social. Colegios, centros de salud, canchas deportivas, entre otras infraestructuras, fueron comprendidas como parte del problema habitacional (Palma y Sanfuentes, 1979). La CORVI, en tanto, pasó a ser una institución dependiente de este ministerio y limitó sus responsabilidades a la construcción de las viviendas públicas.

El programa de vivienda entre 1964 y 1970 apuntaba a construir sesenta mil unidades por año, es decir, 360 mil viviendas definitivas (Haramoto, 1980). A mediados de los sesenta la familia chilena promedio era de 5,4 personas, es decir, si se quería terminar con la crisis en vivienda, se necesitaba construir una ciudad para cerca de dos millones de personas en un periodo de seis años. De estas viviendas, 213 mil correspondían a sectores de bajos recursos. La situación empeoró tras las fuertes tormentas de 1965 que tuvieron devastadoras consecuencias en la zona central: la presión social por vivienda creció fuertemente y se hacían necesarias medidas fuera de las tradicionales para dar respuesta al problema de la escasez, en un corto espacio de tiempo y con un presupuesto acotado.

DE LA OPERACIÓN SITIO A LA OPERACIÓN TIZA

La "Operación Sitio" surgió en 1965 como una política de emergencia ante a los daños causados por los temporales, pero tan solo dos años después, a través

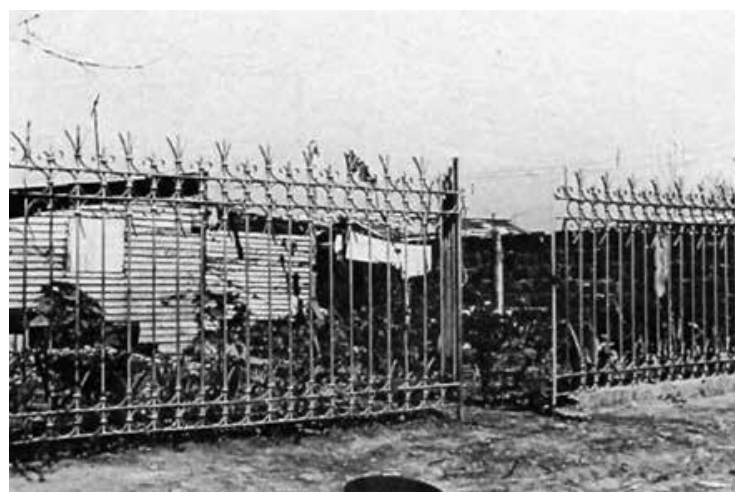

FIG 12 del Plan de Ahorro Popular, se convertiría en una vía formal para dotar de acceso a la vivienda a la población de menores ingresos. El programa consistía básicamente en la entrega de créditos para la compra de lotes unifamiliares, urbanizados y conectados a la ciudad, donde los pobladores autoconstruirían sus viviendas. Siguiendo los postulados del MINVU, estos conjuntos se planificarían con el debido equipamiento educacional, recreativo y de salud. Entre los años 1965 y 1970, cerca de 71 mil sitios fueron entregados, beneficiando a más de 380 mil personas (Garcés, 2002, p. 308). Sin embargo, la calidad de los sitios urbanizados era dispar, con lo que pobladores y opositores a esta política pública la comenzaron a llamar "Operación Tiza”, dado que en algunos casos las entregas no superaban más que un lote trazado con ese material.

Hacia 1962 los informes de la CORVI indicaban que un $76 \%$ de la inversión en vivienda definitiva se gastaba en la construcción de las edificaciones, mientras que el terreno significaba un $5 \%$ y el costo de la urbanización un $19 \%$ de la inversión total (CORVI, 1962; CORVI, 1963). De esta manera, el proceso de autoconstrucción reduciría la inversión inicial, permitiendo entregar una solución a los pobladores de escasos recursos que no tenían la posibilidad de lograr el ahorro mínimo para acceder a una vivienda definitiva por la vía tradicional. La "Operación Sitio", por tanto, permitía acelerar los procesos de distribución de terrenos, masificando el acceso a la vivienda a través de la construcción incremental por parte de sus pobladores.

El Plan de Ahorro Popular consistía en cinco opciones ${ }^{3}$, siendo la primera y más básica la correspondiente a la "Operación Sitio". Se trataba en una primera etapa de la entrega de un lote de 160 $\mathrm{m}^{2}$ que, en el mejor de los casos, podría tener una mediagua ${ }^{4}$ ubicada al fondo del lote. La urbanización se reducía a calles ripiadas y soleras, redes de agua potable en pilones y redes eléctricas de distribución y alumbrado. El equipamiento se definía como una escuela, centros comunitarios, locales comerciales y áreas libres. En la segunda etapa se completaría la urbanización con la instalación de servicios de alcantarillado, agua potable y electricidad. La construcción de la vivienda definitiva sería de responsabilidad del poblador (Labadía, 1970).

La CORVI cumplía con la función de proveer los terrenos necesarios para desarrollar las operaciones.

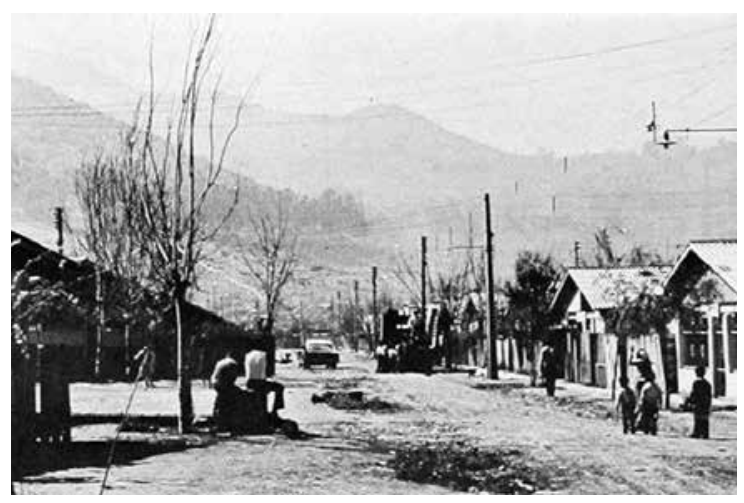

FIG 13
Las cinco opciones del Plan de 3 Ahorro Popular correspondían a (1) sitios semiurbanizados, (2) sitios con urbanización completa, (3) viviendas básicas de autoconstrucción, (4) viviendas construidas de $45 \mathrm{~m} 2$ de un piso, $\mathrm{y}(5)$ departamentos en edificios de 4 pisos (Labadía, 1970, p. 429)

Estructura monolocal de madera de $18 \quad 4$ m2, usada comúnmente en Chile como vivienda de emergencia.

El libro El lote $9 \times 18$ en la encrucijada 5 habitacional de hoy, de Montserrat Palmer Francisco Vergara, presenta una acabada investigación arquitectónica de las urbanizaciones realizadas por las poblaciones
construidas en Santiago entre 1959 y 1988. 
Las poblaciones construidas entre 1966 y 1970 se distribuyeron de forma homogénea en las comunas periféricas de la capital, en su mayoría en torno al anillo de Américo de Vespucio, vía intercomunal que para entonces hacía posible esta expansión de la ciudad (Palmer y Vergara, 1990) (fig. 7). En estos conjuntos el diseño urbano se redujo al mínimo para lograr la mayor eficiencia económica. El objetivo era distribuir la mayor cantidad de lotes y espacios públicos en la menor área posible, maximizando además la cantidad de sitios que serían servidos por las calles que enfrentan. De esta forma la mayor eficiencia se lograba a través de un lote rectangular que tuviese el menor frente posible hacia la calle, para que un mayor número de casas se pudiesen conectar a las redes de alcantarillado y electricidad, disminuyendo la inversión en construcción de metros lineales de urbanización (fig. 8-13).5

Algo relevante de la "Operación Sitio" es que no consideraba solo la construcción de viviendas, sino que incluía -al menos en plan- la construcción de colegios, centros de salud, áreas deportivas, espacio público y zonas comerciales, entre otras infraestructuras. El sistema de prefabricación se ensayaría para la construcción de escuelas, mientras que el Ministerio de Educación estudiaría la dotación de profesores (Garcés, 2002). El mayor problema fue que el prometido equipamiento no fue construido en todos los casos (Hidalgo, 2005). No será sino hasta la década de los ochenta y en adelante, que los distintos gobiernos mejorarán paulatinamente las condiciones urbanas en que se encontraban estos conjuntos.

La “Operación Sitio" recibió severas críticas no solo porque el proceso de autoconstrucción se consideraba como discriminatorio hacia la gente con menos recursos, sino también por su localización. Autores como Manuel Castells (1971) argumentaban, ya a principios de los setenta, que este era el comienzo de una masiva segregación social. Este hecho se incrementó exponencialmente dadas las políticas públicas adoptadas en las siguientes décadas y que continuaron la expansión de las operaciones Sitio a través de paños socialmente homogéneos y desprovistos -en muchos casos hasta el día de hoy-de los equipamientos, infraestructuras y servicios mínimos para el desarrollo de las personas: educación, salud, empleo, comercio y áreas verdes, entre otros.

\section{UN CASO EJEMPLAR}

La Villa La Reina ${ }^{6}$ es un barrio de 1.592 casas construido completamente por sus pobladores: desde las viviendas hasta las áreas verdes, colegios, iglesias y espacios públicos, incluyendo calles, redes de alcantarillado y agua potable. Es de los mayores programas de autoconstrucción asistida de la década del sesenta, no solo en Chile sino en todos los países en desarrollo. El trabajo colaborativo entre la alcaldía, los pobladores y la Universidad Católica, diferencia a la Villa La Reina de los conjuntos construidos por la "Operación Sitio" o por posteriores programas como el de Sitios y Servicios? que, desarrollados por gobiernos nacionales, se alejaban de los problemas locales de las comunidades. En esta tarea colaborativa fue relevante la participación del arquitecto Fernando Castillo Velasco, quien siendo alcalde de la Municipalidad de La Reina, profesor y posterior rector de la Universidad Católica, logró convocar a instituciones políticas y académicas con las necesidades y expectativas de una comunidad de pobladores que se organizaba para obtener su derecho a la vivienda.

\section{LA CREACIÓN DE LA COMUNA DE LA REINA: TENSIONES SOCIALES Y SEGREGACIÓN}

Durante la década de 1950 la comuna de Ñuñoa crecía rápidamente con la llegada de diversas clases sociales, en su mayoría provenientes de sectores de ingresos medios y altos. Su cercanía a las comunas centrales de Santiago, donde se concentraban las mayores fuentes de empleo y comercio, atraía a la población a construir sus viviendas en esta zona. Por contraparte y, rápidamente, comenzaron a surgir áreas de industrias y servicios, proliferando de esta manera el empleo y atrayendo a esta comuna a pobladores de escasos recursos quienes comenzaron a habitar, ilegalmente, terrenos públicos o privados.

Con el tiempo, la tensión social entre los distintos vecinos de la comuna de Ñuñoa comenzó a incrementar. Los sectores socioeconómicos altos y medios, que en su mayoría habitaban el área precordillerana, comenzaron a exigir un trato distinto por parte de la alcaldía. Sus exigencias escalaron hasta la presidencia de Alessandri, quien en 1963 decidió separar definitivamente este sector del resto de la comuna de Nuñoa, fundando la Municipalidad de La Reina. Este no fue el único acto de segregación. Óscar Castro, primer alcalde de la comuna, tramitó un decreto por el cual se cercarían los terrenos ocupados por habitantes ilegales, forzando su salida y evitando que nuevas tomas se produjeran en la comuna (San Martín, 1988).

En 1964 el presidente Eduardo Frei Montalva designó como alcalde de La Reina a Fernando Castillo Velasco, también militante del partido de la Democracia Cristiana. Las primeras acciones de su alcaldía tenían por objetivo, por una parte, eliminar la segregación impuesta en los años anteriores y, por otra, promover un desarrollo social para la comuna. Es así como derogó el decreto impuesto por Castro y comenzó a elaborar un Plan de Desarrollo Comunal en conjunto con la Universidad Católica ${ }^{8}$. Fernando Castillo estableció durante su administración las bases para un desarrollo inclusivo e integral siendo partícipes todos los niveles socioeconómicos, incorporando actividades industriales, institucionales y culturales en una zona de la ciudad que en principio se perfilaba como una comuna dormitorio. En sus propias palabras, "un día me encontré que había 1.600 familias que no tenían casa, que vivían a orillas del canal San Carlos, en sitios baldíos. Los convoqué y les dije ustedes son vecinos de la comuna, tienen los derechos de cualquier vecino; era gente de extrema pobreza, y les dije que yo me comprometía a hacer todo lo posible por que ellos fuesen propietarios" (Cociña, Quintana, Valenzuela, 2009, p. 127). De esta forma, 
FIG 14

Planta de la Villa La Reina/Plan of the Villa La Reina.

E./Sc. 1: 10.000 .

Fuente/Source: Haramoto, 1985, p. 87.

Redibujo del autor/Redrawn by the author.

1. Plaza/Plaza

2. Comercio/Commerce

3. Escuela/School

4. Plazoletas interiores/Interior plazas

5. Mercado/Market

6. Capilla/Chape

\section{FIG 15}

Colegio Villa La Reina, c. 1967/Villa La Reina School, c. 1967.

Fuente/Source: Alvarado, 1967, p. 34.

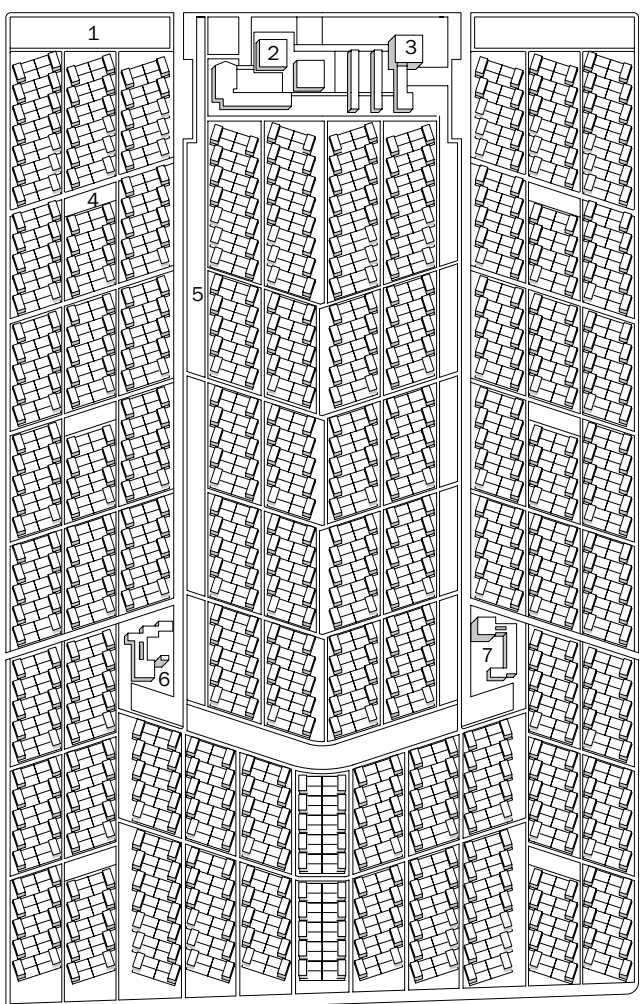

FIG 14

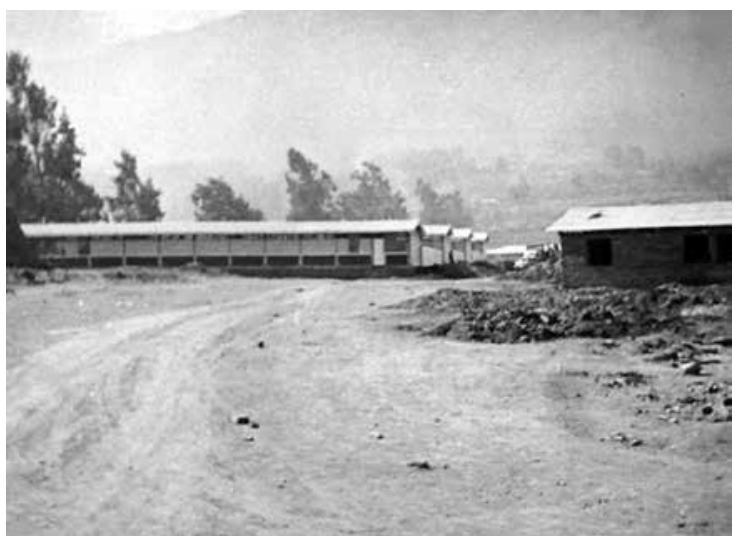

FIG 15 los pobladores que habitaban ilegalmente paisajes degradados de la comuna, pudieron acceder a una casa. La incorporación de vivienda popular dentro del plan de desarrollo fue fundamental para la incorporación de otros usos de suelo en el sector (Castillo, 2013).

La construcción de Villa La Reina surgió como una vía para que la gente en extrema pobreza pudiese continuar viviendo en la comuna y no fueran erradicados a lugares distantes donde perderían las redes sociales y de trabajo que habían creado durante los últimos años. Así, el primer objetivo de la alcaldía era encontrar un terreno asequible para la municipalidad donde poder radicar a los pobladores. Por otra parte, dada la escasez de recursos, la autoconstrucción a través de la organización de la comunidad local aparecía como una solución factible para reducir los costos de inversión.

\section{LOCALIZACIÓN: CONEXIÓN CON LOS RECURSOS DE LA CIUDAD}

El área seleccionada para implementar parte del Plan de Desarrollo Comunal y la consecuente construcción de la villa fue el fundo La Reina que se encontraba en el centro de la comuna. El terreno, una antigua zona agrícola prácticamente inactiva, contaba con 220 ha pertenecientes al Servicio de Seguro Social. Fernando Castillo, luego de reuniones con distintas instituciones y políticos, entre ellos el entonces senador Salvador Allende, logró adquirir los terrenos a un bajo costo (Castillo Velasco, 2008, p. 25; Cociña, Quintana, Valenzuela, 2009, p. 127). Se especificaba en la compra que un $20 \%$ del total debía destinarse a los pobladores en situación inestable (Alvarado, 1967).

El Plan de Desarrollo Comunal establecía la construcción de un parque industrial constituido por cerca de cien industrias ligeras y pequeñas empresas compatibles con el desarrollo de viviendas (Eliash, 1990). De esta forma, la Villa La Reina se ubicaría vecina a una fuente de empleo que podría dar trabajo al menos a la primera generación de habitantes del conjunto. El acceso de los pobladores a esta nueva fuente de trabajo reduciría de manera importante una de las mayores inequidades urbanas de las que sufren los desarrollos tradicionales de vivienda social, tanto en Chile como en el mundo.

Si bien La Reina es una comuna periférica dentro de Santiago, la villa se ubicó junto a Av. Larraín ${ }^{9}$, vía intercomunal que conecta al municipio con el anillo vial de Américo Vespucio y con las comunas más céntricas de la capital. Por otra parte, con el paso del tiempo y gracias a la debida planificación, este sector se ha convertido en un subcentro comunal anexando múltiples equipamientos culturales, deportivos, educacionales, institucionales y de salud. Sumado a esta variedad programática, la comuna ha mantenido una diversidad socioeconómica que la diferencia de los extensos paños aislados y socialmente homogéneos que se crearon por el abuso de la "Operación Sitio" en comunas como Pudahuel,

Especificamente la Villa La Reina se ubica entre las calles Larraín, Diputada Laura Rodríguez, Talinay y Cordillera. en los terrenos que actualmente conforman la comuna de Cerro Navia. 


\section{FIG 16}

Viviendas de la Villa La Reina, c. 1967/

Homes in Villa La Reina, c. 1967.

Fuente/Source: Alvarado, 1967, p. 36

\section{FIG 17}

Planta y elevación viviendas de la Villa La Reina/Plan and elevation of Villa La Reina housing.

E./Sc. 1: 500

A la izquierda del plano la vivienda original. A la derecha la ampliación propuesta en el fondo del sitio/To the left, the original housing plan. To the right, the proposed on-site expansion.

Fuente/Source: Haramoto, 1985, p. 87. Redibujado por el autor/Redrawn by the author.

\section{FIG 18}

Fernando Castillo Velasco durante la construcción de las viviendas/

Fernando Castillo Velasco during housing construction.

Fuente/Source: Castillo Velasco, 2008 p. 32

\section{FIG 19}

Fabricación de los ladrillos con tierra del lugar, c. 1967/On-site brick manufacture, c. 1967.

Fuente/Source: Alvarado, 1967, p. 39.

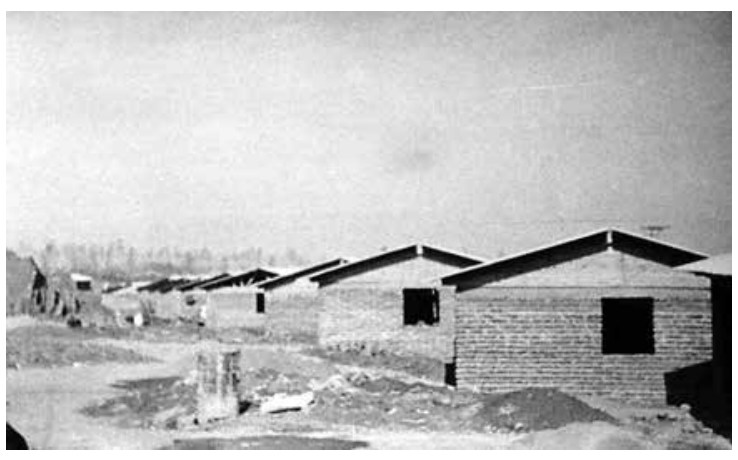

FIG 16
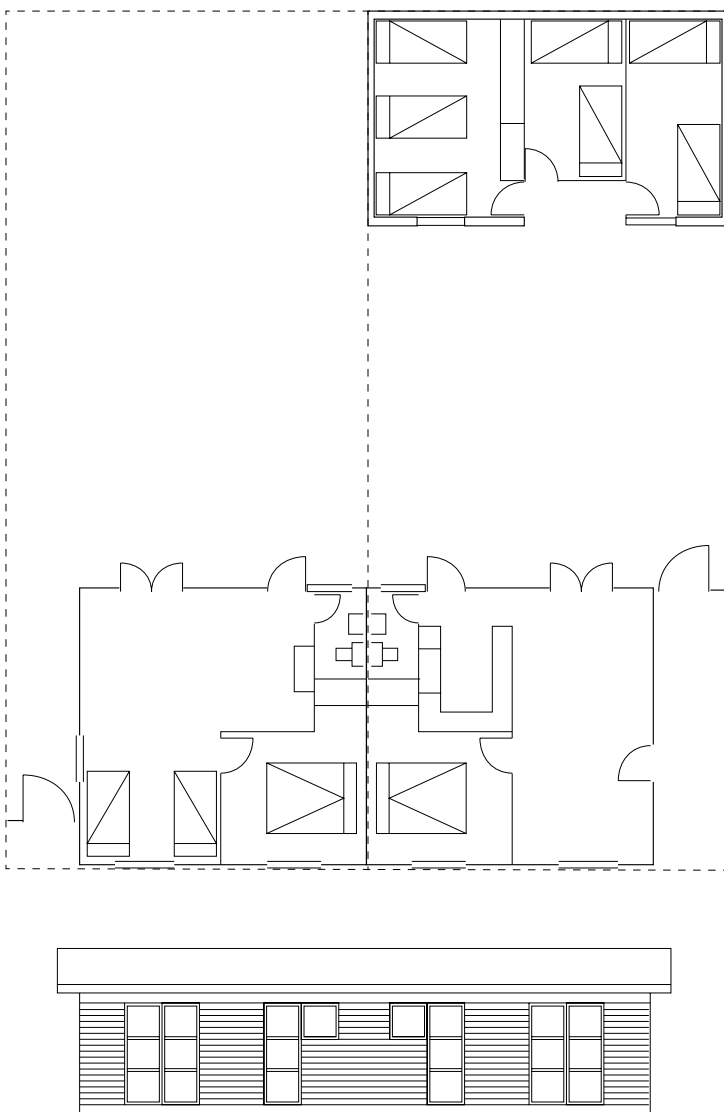

FIG 17

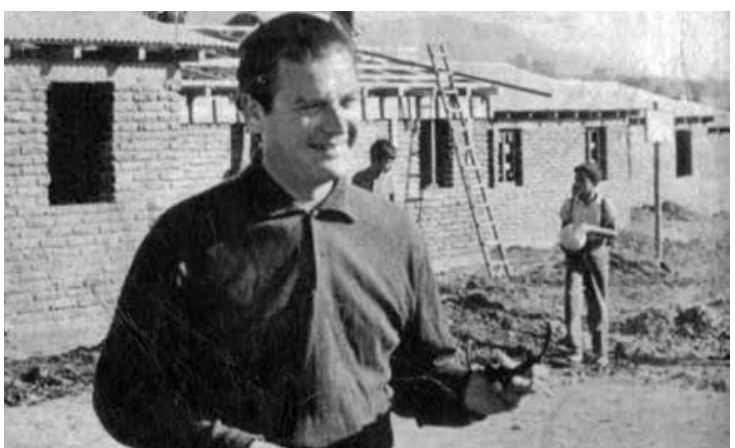

FIG 18

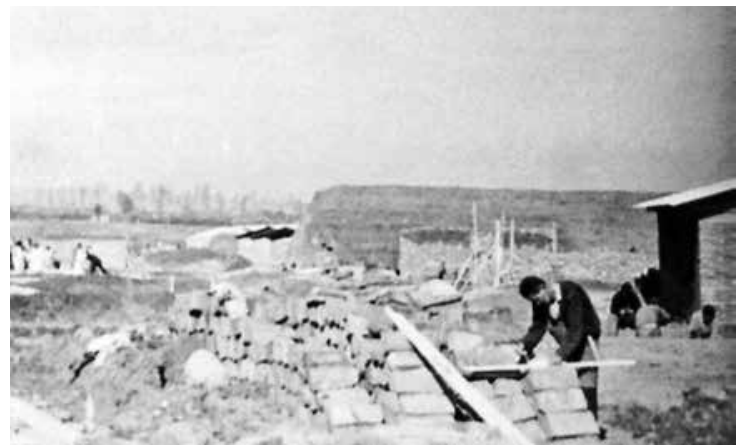

FIG 19

DISEÑO URBANO:

LAS BASES PARA LA CONSTRUCCIÓN INCREMENTAL DE UN BARRIO.

El diseño participativo realizado por profesores y alumnos de la Escuela de Arquitectura de la Universidad Católica con los futuros pobladores de Villa La Reina, logró introducir mejoras a un diseño urbano que perseguía los mismos objetivos de eficiencia económica que aquellos desarrollados por la "Operación Sitio" (fig. 14). En este caso no se trató de un diseño urbano dedicado exclusivamente a la distribución eficiente de lotes, sino también de una adecuada distribución y diseño de calles, pasajes peatonales, bulevares, plazas y la designación de los terrenos para zonas comerciales, colegios y mercados, entre otros equipamientos. Uno de los objetivos de los talleres académicos realizados por los profesores Fernando Castillo Velasco y Mario Pérez de Arce Lavín con los pobladores, era que el diseño de la villa no tuviera los problemas de las poblaciones de la época, sino que fuera "un barrio más", integrado con la ciudad (San Martín, 1992; Castillo, 2013).El resultado de estos talleres fue continuado por el arquitecto Renato Parada. Relevante en los procesos de gestión, diseño y construcción es la figura del arquitecto Eduardo San Martín, Director de Obras de la Municipalidad durante esos años. 

futuras casas, c. 1967/Men and women building their future homes, c. 1967. Fuente/Source: Alvarado, 1967.

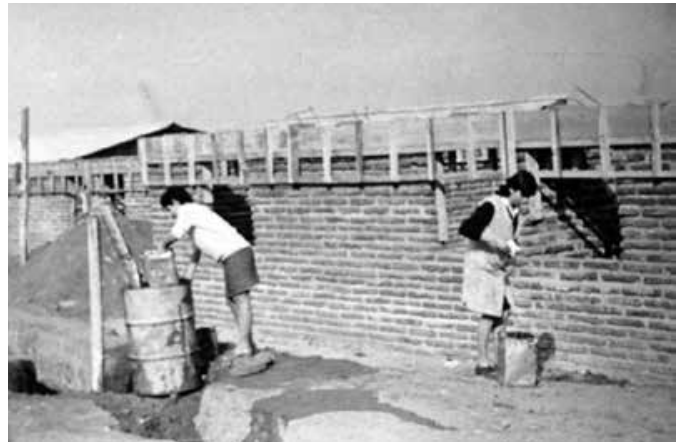

FIG 20
Villa La Reina no estaría constituida solo por casas, sino que contaría además, entre otros equipamientos, con un colegio, construido el primer año (fig. 15) y zonas comerciales. Estos espacios comunitarios se ubicaron contiguos a Av. Larraín, principal calle de la comuna, como puertas de acceso a la villa, con el objetivo de tener una mejor relación entre el barrio y el contexto urbano inmediato de la ciudad (San Martín, 1988). Otros equipamientos como mercados e iglesia, también autoconstruida por los pobladores, se ubican al interior de la villa junto a su calle principal.

En términos de espacio público, arquitectos y pobladores deciden evitar grandes plazas cuadradas, las que generalmente se convierten en espacios deteriorados y focos de delincuencia. Al contrario, se introducen pequeñas plazoletas distribuidas junto a casas y pasajes peatonales al interior de la villa. $\mathrm{La}$ principal área verde sería una ancha vereda arbolada que se ubica a lo largo de la calle principal del barrio, la que concentra el transporte urbano y los mayores costos de urbanización (San Martín, 1988). Esta faja verde distancia a las casas de la calle, mientras que su ancho es posible de ser mantenido por los propios vecinos. Estos espacios públicos son relevantes dado que son las expansiones naturales de las viviendas, facilitando además la interacción de sus habitantes. La cohesión social y la identidad urbana son relevantes en el cuidado y progreso del barrio.

La definición de las viviendas y su distribución son parte del resultado de los talleres académicos y del trabajo de pobladores y arquitectos de la municipalidad. Los primeros dos diseños son rechazados por la comunidad por ser casas demasiado pequeñas (Alvarado, 1967). Los bloques continuos con viviendas pareadas, similares a los de

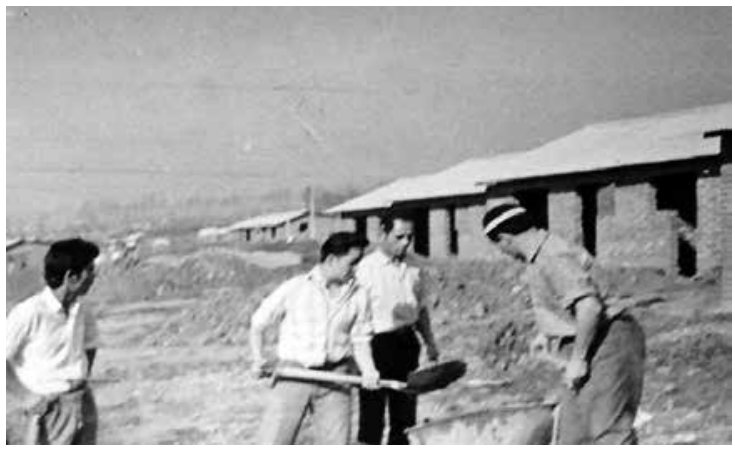

FIG 21

la Operación Sitio, tampoco son aceptados dado que los pobladores aspiraban a casas individuales. Por este motivo es que los lotes están girados respecto de las calles que enfrentan (fig. 16), con el objetivo de traslapar y separar unas viviendas de otras (Castillo, 2013). Las casas se definieron en $36 \mathrm{~m}^{2}$, con dos dormitorios, un estar-comedor, cocina y baño. Los lotes (fig. 17) de $165 \mathrm{~m}^{2}$, serían de 7,5 metros de frente por 22 metros de fondo (Alvarado, 1967). Las casas se construirían al frente del sitio ocupando casi todo su ancho, con el objetivo de mantener un cierto orden construido en la fachada que da a la calle. El patio trasero, en tanto, se plantea como un espacio disponible para futuras ampliaciones.

\section{AUTOCONSTRUCCIÓN: \\ ORGANIZACIÓN Y DESARROLLO SOCIAL}

A comienzos de 1965 los pobladores comienzan a organizarse en la que será la "Federación de Pobladores", formada por 1.600 familias reunidas en 16 comités, quienes están a cargo de la urbanización y construcción de sus propias casas. Colectivamente definen sus estatutos, estableciendo, entre otras cosas, que una o dos personas por familia trabajarían solamente en días sábados, domingos y festivos, de modo de no afectar los empleos regulares de las personas. Las distintas familias trabajarán sin saber cuál será su vivienda definitiva hasta que el conjunto esté terminado. La distribución de casas se hará por medio de puntajes que las familias van acumulando según su desempeño en la construcción y administración de la obra. La asistencia técnica es provista en un principio por la Municipalidad y luego se suma el instituto de formación técnica INACAP; alumnos de Construcción Civil de la Universidad Católica colaboran con el seguimiento de la obra. En mayo de 1966 los pobladores construyen la casa piloto y en agosto del mismo año comienzan la edificación de todo el conjunto (Alvarado, 1967).

El proceso de autoconstrucción se planteó tanto como una oportunidad para que los pobladores puedan aprender nuevas herramientas de trabajo, como también para la conformación de empresas gestionadas por los propios vecinos. De esta forma, INACAP realizó semanalmente cursos de capacitación para jefes de obra y ayudantes. Las autoridades municipales, por su parte, fomentaron que la comunidad estableciera sus propias industrias y así comenzó la instalación de fábricas de ventanas y puertas, planchas de yeso, tubos de alcantarillado, pavimentos prefabricados y armado de cerchas. Con la tierra del lugar se fabricaron sus

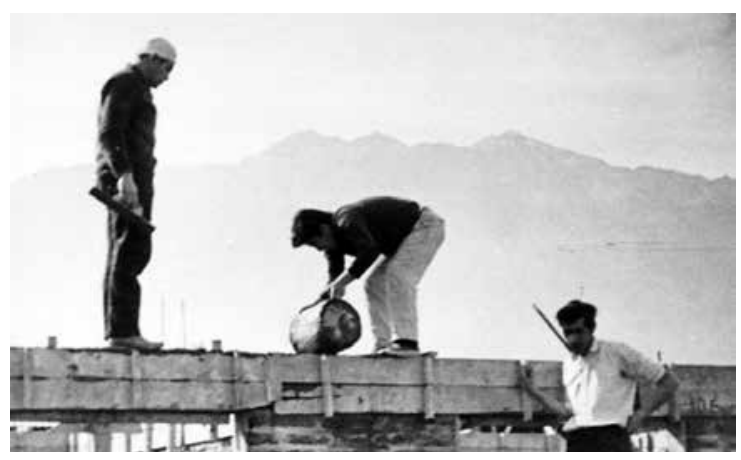

FIG 22

propios ladrillos (fig. 18-22). Si bien son empresas que surgen para construir sus propias casas y urbanizar su barrio, producen excedentes de materiales que venden a otros conjuntos de vivienda de Santiago, a la vez que se involucran en la construcción de otras obras de la comuna como bancos y supermercados, e incluso el Parque Industrial vecino a la villa (Alvarado, 1967; Haramoto, 1980; San Martín, 1988; San Martín, 1992).

La autoconstrucción se establece en Villa La Reina como una forma de crear sentido de pertenencia y compromiso con el proyecto, además de asentar las confianzas de los pobladores en sus propias capacidades (Márquez, 2006). Si bien surgió como una estrategia técnica para reducir los costos de inversión, 


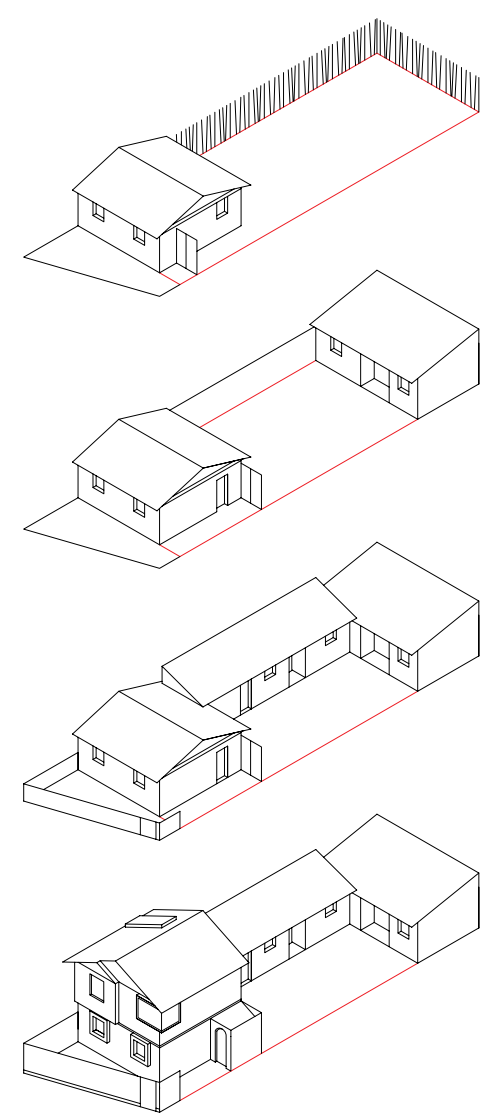

FIG 23

Esquema de ampliación de las viviendas de Villa La Reina, desde la casa original hasta el cierre del antejardín y ampliaciones en e patio y en segundo piso/Expansion scheme of the Villa la Reina dwellings, from the original house to the enclosure of the front yard and expansions in the patio and second floor.

s/ E/Not to scale.

Fuente: dibujo del autor/Source: Author's drawing.

\section{FIG 24}

Viviendas originales sin ampliaciones, 2014/Original houses without additions, 2014.

Fotografía/Photography: Stephannie Fell.

\section{FIG 25}

Viviendas ampliadas en segundos pisos 2014/Second-floor add-ons, 2014. Fotografía/Photography: Stephannie Fell.

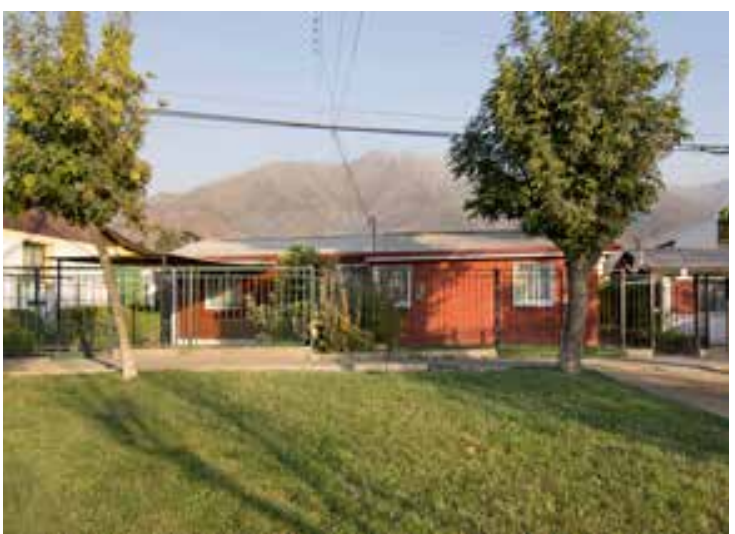

FIG 24

se convirtió en una vía de integración social de los pobladores de escasos recursos tanto a la economía nacional como a los ámbitos de decisiones políticas para el desarrollo de su entorno social y construido, superando de esta forma la marginalidad en que se encontraban (San Martín, 1992; Castillo Velasco, 2008; Zerán, 1998).

\section{EL DERECHO A LA VIVIENDA DIGNA. GOBIERNO DE SALVADOR ALLENDE}

Salvador Allende, al igual que Eduardo Frei Montalva, estableció durante su gobierno (1970-1973) que la vivienda sería un derecho irrenunciable de la población, pero a diferencia de la administración anterior, agregó que "es obligación del Estado proporcionar vivienda a su Pueblo y ella no puede ser objeto de lucro" (Haramoto, 198o, p. 33). La nueva postura del gobierno apuntaba a la entrega de viviendas definitivas, eliminando de esta forma los programas de autoconstrucción y de vivienda incremental. La "Operación Sitio" fue criticada durante este periodo argumentando la reducida calidad de la solución entregada y la deficiente participación de los afectados en los procesos de diseño y gestión de sus viviendas (Hidalgo, 2005). La autoconstrucción, por su parte, era entendida como un retraso en la ejecución de las

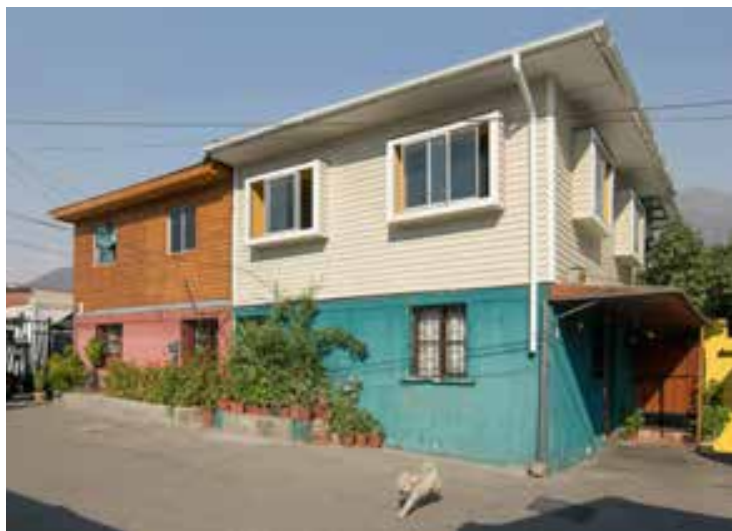

FIG 25

obras e ineficiente en el uso de los recursos económicos (Palma y Sanfuentes, 1979).

Las nuevas políticas adoptadas por el gobierno socialista debilitaron el apoyo estatal a Villa La Reina, a la vez que las dinámicas sociales se fragmentaron y politizaron (Márquez, 2006). Sin embargo, los trabajos de autoconstrucción en la villa no se detuvieron, en gran medida, gracias a lo avanzado que se encontraba el conjunto y a que las organizaciones sociales no quisieron modificar su estructura de trabajo (San Martín, 1988).

Los asentamientos informales continuaron aumentando en la capital, a pesar de los esfuerzos del gobierno de Salvador Allende por reducir la crisis en vivienda. Las tomas ya se venían incrementando desde el gobierno de Frei, pasando de cuatro tomas en 1968 a 104 en 1970, con una población aproximada de 350 mil personas. Para 1973, la cantidad de gente viviendo en campamentos llegó a quinientos mil (De Ramón, 1990).

\section{LA VIVIENDA COMO BIEN DE CONSUMO.} DICTADURA DE AUGUSTO PINOCHET (1973-1990)

Con la llegada de Augusto Pinochet al poder, la vivienda deja de ser un derecho irrenunciable de las personas y pasa a ser "un derecho que se adquiere con

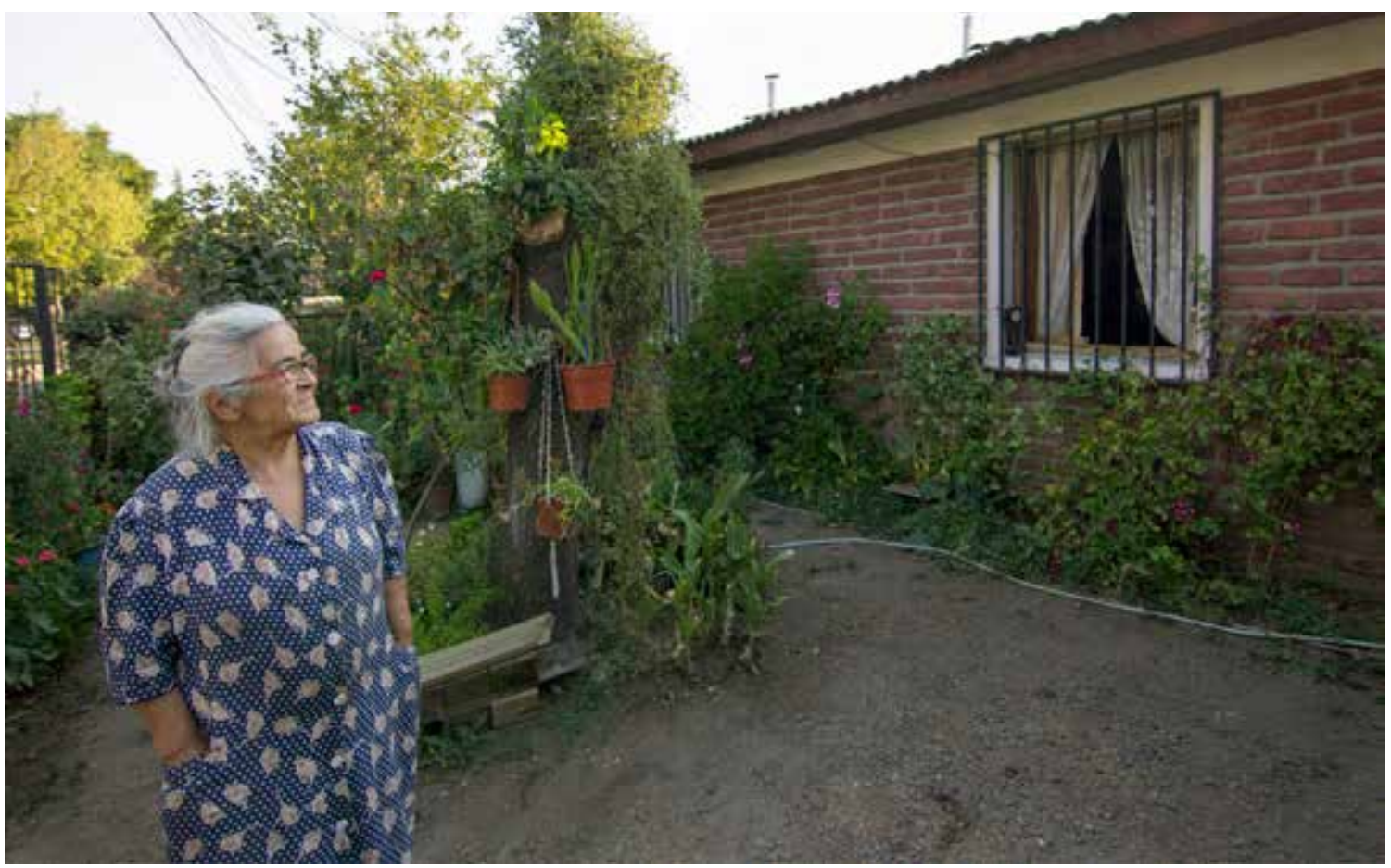




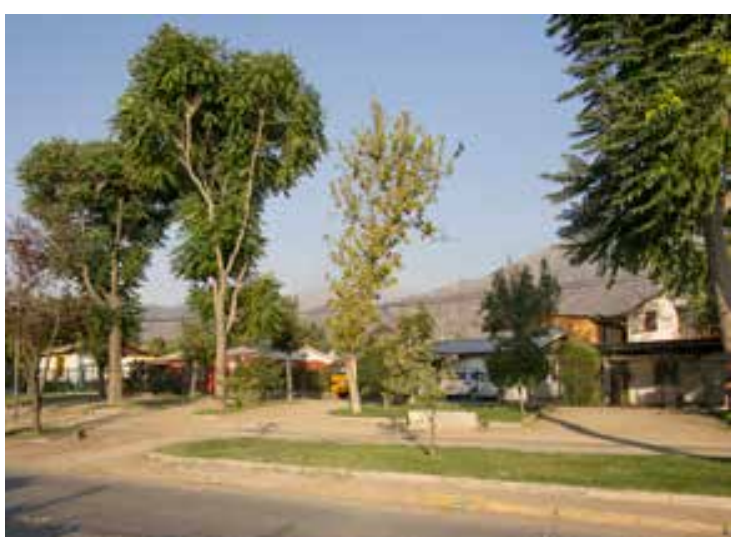

FIG 26

el esfuerzo y el ahorro" (Haramoto, 1988, p. 35). En los setenta y ochenta, y a medida que se adopta un modelo económico neoliberal, el Estado comienza a cumplir un rol subsidiario respecto de la vivienda social y son empresas privadas las encargadas de encontrar los terrenos para realizar las nuevas construcciones. Bajo esta lógica es que en 1979 se liberaliza el mercado de suelos, argumentando que con el aumento en la cantidad de terrenos disponibles disminuirían los precios y se haría factible la construcción de más viviendas sociales, hecho que no ocurrió (Hidalgo, 2005).

La segregación social que había germinado con las operaciones Sitio fue incrementada fuertemente luego de la liberalización del mercado de tierras. La especulación que trajo esta medida aumentó considerablemente el valor de los suelos céntricos de la capital, afectando particularmente a las poblaciones informales que se ubicaban en estos terrenos. Se implementó una política de erradicación de campamentos que desplazó a estas comunidades desde el centro de la ciudad hacia la periferia, concentrando la pobreza en comunas que no contaban con los equipamientos de salud, educación o empleo necesarios para el desarrollo. Entre 1979 y 1985 se relocalizaron más de 170 mil personas, expandiendo Santiago a un ritmo de 3.0oo ha anualmente

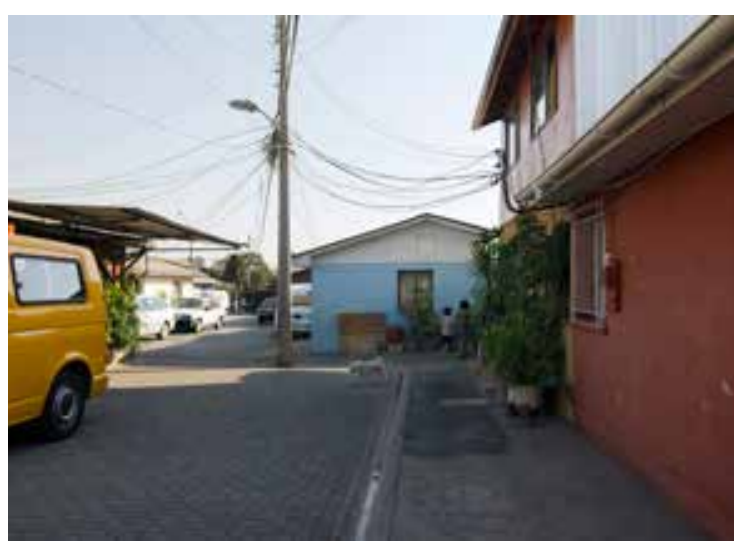

FIG 27

(Hidalgo, 2004). Durante este tiempo los dirigentes de Villa La Reina fueron perseguidos, las industrias vecinales clausuradas y las organizaciones disueltas (San Martín, 1988; Márquez, 2006). Las pocas casas que quedaban por construir hacia 1973, ubicadas al oriente del conjunto, fueron terminadas individualmente por los pobladores con algo de apoyo de la administración (Castillo, 2013, p. 98).

\section{LA INFORMALIDAD ESCONDIDA}

Las migraciones a la capital en búsqueda de trabajo y oportunidades educacionales seguían creciendo durante las décadas de 1970 y 1980. La formación de nuevas poblaciones informales estaba prohibida y las ciudades eran severamente controladas por militares. La gente, ante la escasez de viviendas sociales y ante la imposibilidad de establecer campamentos, comenzó a densificar las pequeñas soluciones habitacionales provistas en años anteriores por las operaciones Sitio y demás políticas habitacionales. Una familia podía recibir en su propiedad a otras dos o tres familias como allegados. Comenzó una fuerte densificación de los barrios de escasos recursos. La descrita segregación social seguía aumentando, ahora por hacinamiento, y las necesarias infraestructuras o conexiones con los recursos de la ciudad seguían sin ser construidos.

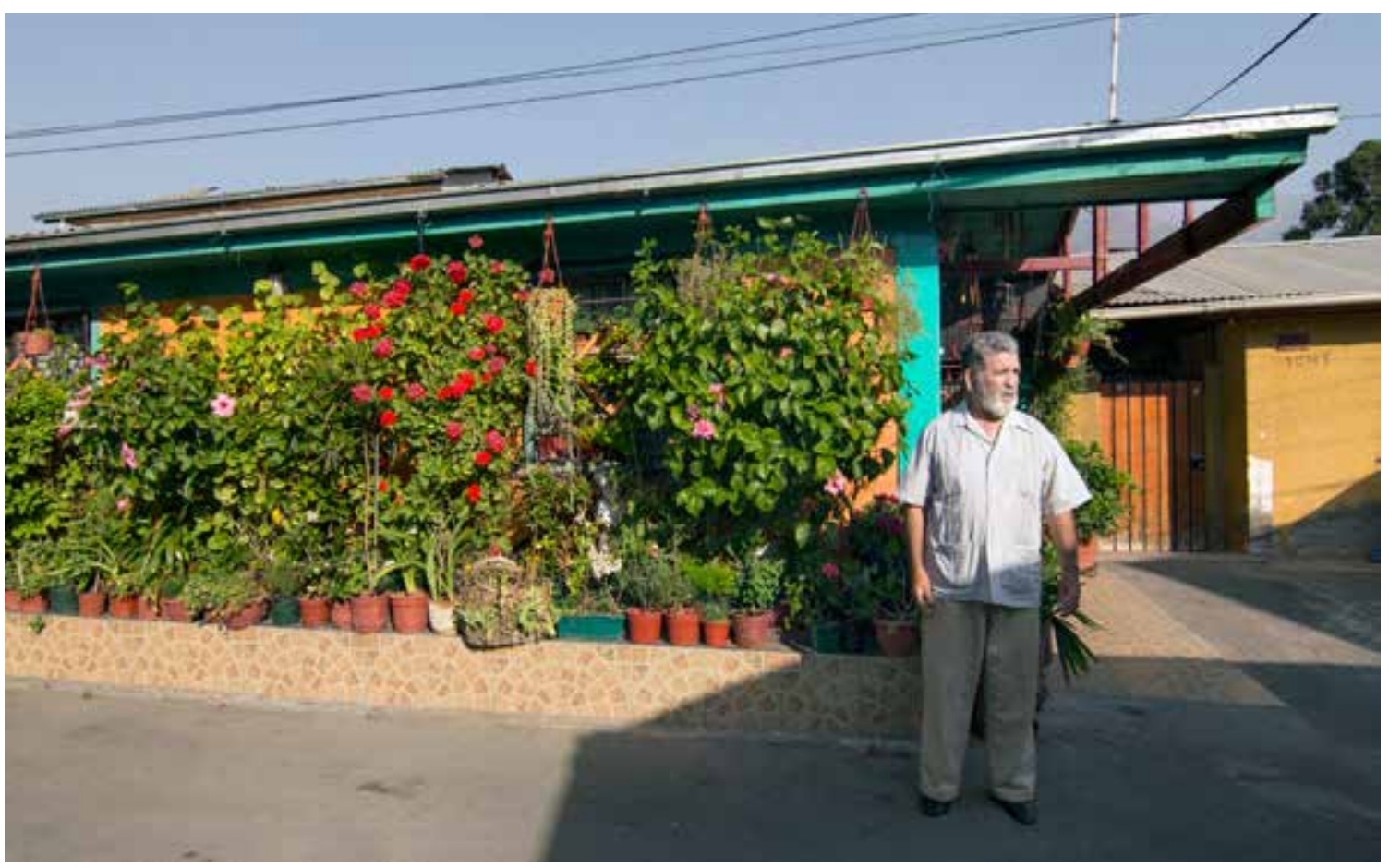


Villa La Reina no fue la excepción a este proceso de densificación. Con el tiempo se comenzaron a construir ampliaciones de material ligero en los patios traseros, las que han ido mejorando y se han hecho permanentes. De esta forma y con el paso de los años, los pobladores originales han ido recibiendo a las familias de sus hijos y otros allegados (Castillo, 2013) (fig. 23). Los datos socioeconómicos muestran que en Villa La Reina, hacia el año 2002, cerca de un $40 \%$ de los pobladores que seguían viviendo en la villa habían dejado la extrema pobreza para ser parte de la clase media. De acuerdo a lo investigado por Francisca Márquez, entre los años 2006 y 2009, a partir de las segundas generaciones se han observado procesos de movilidad social y profesionalización, pero quisieron permanecer viviendo en la misma comuna (fig. 24-29).

\section{URBANIZANDO CON TIZA}

Ante la urgencia y falta de recursos, la estrategia tras la "Operación Sitio" y Villa La Reina buscaba resolver la crisis de vivienda entregando a las personas lo básico que no podrían obtener por sí solas. La población viviendo en extrema pobreza y de forma ilegal sería dueña de un terreno y, ya sin el temor de ser expulsada, podría mejorar progresivamente tanto su vivienda como su entorno urbano. Si el sitio le era entregado donde ya habitaba, podía mantener las redes sociales y de empleo que había generado anteriormente. El acceso a los servicios básicos de agua potable y alcanta- rillado mejoraría la calidad de vida de los pobladores. El diseño urbano, por sencillo que fuese, mantendría un cierto orden en el desarrollo de los conjuntos, evitando la ineficiencia, insalubridad e inseguridad que suelen tener los asentamientos informales que se generan de forma espontánea (United Nations Human, 2003). Se planificaría además la construcción de equipamientos comunitarios como colegios, canchas deportivas, áreas verdes, zonas comerciales y centros de salud, entre otros, que promoverían el desarrollo social. El terreno, al estar inserto y conectado con la ciudad, otorgaría la posibilidad a los pobladores de acceder a las oportunidades de empleo que se generan en los centros urbanos. El proceso de autoconstrucción dotaría de nuevas herramientas profesionales a la población, a la vez que el trabajo colectivo fomentaría la creación de una identidad de barrio, de nuevas redes sociales y de ayuda mutua.

Las grandes migraciones urbanas siguen siendo un problema hoy en día, tal vez no en Chile, pero sí en el resto del mundo. En la última década las ciudades en Asia, África y Latinoamérica sumaron, respectivamente, 880 mil, 230 mil y 150 mil nuevos habitantes semanalmente (United Nations, 2012, p. 29). Estrategias similares a las de la "Operación Sitio" se continúan implementando en países como Paquistán e India para evitar la formación de poblaciones informales. Es de esperar que se repliquen los aciertos y no los errores. ARQ
FRANCISCO QUINTANA | Arquitecto y Magíster en Arquitectura, Pontificia Universidad Católica de Chile, 2010; Master in Design Studies: Urbanism, Landscape, Ecology concentration, Harvard University, 2014. Becario Fulbright, 2012-2014. A partir de 2005 su trabajo se ha vinculado a la labor editorial: es fundador y codirector de Cientodiez, BARQO y Volúmenes Independientes y coeditor del libro Agend Pública: Arquitectura > Ciudad > Desarrollo (2009).

\section{REFERENCIAS BIBLIOGRÁFICAS}

ALVARADO, Jorge. Auto Construcción Villa La Reina. Tesis (Constructor Civil). Santiago, Chile, Pontificia Universidad Católica de Chile, Escuela de Construcción Civil, 1967.

CASTELLS, Manuel, et al. Campamentos de Santiago. Movilización Urbana. Milwaukee, University of Wisconsin-Milwaukee, Cente for Latin American Studies, 1971. CASTILLO, María José. Población y gestión habitacional de los pobladores. Articulación con la política de vivienda y barrio. Trayectoria y problemática actual: Tomo 2. Cuadernos de investigación. Tesis (Doctora en Arquitectura). Madrid, España, Universidad Politécnica de Madrid, Escuela Técnica Superior de Arquitectura, 2013.

CASTILLO VELASCO, Fernando. Lecciones del tiempo vivido. Santiago, Catalonia, 2008.

COCIÑA, Camila, QUINTANA, Francisco y VALENZUELA, Nicolás (eds.). Agenda
Pública: Arquitectura $>$ Ciudad $>$ Desarrollo. Santiago, Cientodiez, 2009. CORVI. Segundo Plan Trienal, 1962-1964. Santiago, Corporación de la vivienda, 1962.

CORVI. Plan Habitacional Chile. Santiago, Corporación de la vivienda, 1963. DE RAMÓN, Armando. "La población informal. Poblamiento de la periferia de Santiago de Chile. 1920-1970". EURE [en línea]. Vol. 16, № 50, 1990 [fecha de consulta: 8 de enero de 2014]. Disponible en <http://www. eure.cl/numero/la-poblacion-informalpoblamiento-de-la-periferia-de-santiagode-chile-1920-1970/>

ELIASH, Humberto. Fernando Castillo: de lo moderno a lo real. Bogotá, Editorial Escala, 1990

GARCÉS, Mario. Tomando su sitio: el movimiento de pobladores de Santiago, 1957-1970. Santiago, LOM, 2002. GEISSE, Guillermo. Economía y política de la concentración urbana en Chile. México D.F., PISPAL, 1983.

HARAMOTO, Edwin. "La necesidad de información en el proceso habitacional chileno". AUCA (39), 1980.

HARAMOTO, Edwin et al. "Casos de conjuntos entre 1950/85". CA, (41): 68 107, septiembre de 1985.

HIDALGO, Rodrigo. "La vivienda social en Santiago de Chile en la segunda mitad del siglo XX: Actores relevantes y tendencias espaciales". En: DE MATTOS, Carlos; DUCCI, María Elena "et al". Santiago en la globalización ¿una nueva ciudad? Santiago, Ediciones SUR, 2004. p. 219 241.

HIDALGO, Rodrigo. La vivienda social en
Chile y la construcción del espacio urbano en el Santiago del siglo XX. Santiago, Instituto de Geografía, Pontificia Universidad Católica de Chile, Centro de Investigaciones Diego Barros Arana, 2005.

JÁUREGUI, Jorge Mario. "Urban and Social Articulation: Megacities, Exclusion and Urbanity". En: HERNÁNDEZ, Felipe; KELLETT, Peter William y ALLEN, Lea, (eds.). Rethinking the Informal City: Critical Perspectives from Latin America. Nueva York, Berghahn Books, 2009. p. 207-224. LABADíA, Alberto. "La 'Operación Sitio': una solución habitacional de desarrollo progresivo". Mensaje, (192): 428-432, septiembre de 1970

MÁRQUEZ, Francisca. "Políticas sociales de vivienda en Chile: de la autoconstrucción tutelada a la privatización segregada 1967-1997". Social Policy and the Challenges of Equity and Citizenship, 11 (49): 79-108, marzo de 2006.

MÁRQUEZ, Francisca. "Historias identidades barriales del Gran Santiago: 1950-2000". Avá. Revista de Antropología, (15): 225-242, diciembre de 2009. MORALES, Eduardo y ROJAS, Sergio. Relocalización socio-espacial de la pobreza. Política estatal y presión popular, 1979-1985. Documento de trabajo N²8. Santiago, FLACSO, 1986. PALMA, Eduardo y SANFUENTES, Andrés. "Políticas estatales en condiciones de movilización social: las políticas de vivienda en Chile (1964-1973)". EURE [en línea]. Vol. 6, No 16, 1979 [fecha de consulta: 8 de enero de 2014]. Disponible en: <http://www.eure.cl/numero/ politicas-estatales-en-condiciones-demovilizacion-social-las-politicas-devivienda-en-chile-1964-1973/>. PALMER, Montserrat y VERGARA, Francisco. El Lote 9 × 18: en la encrucijada habitacional de hoy. Santiago, Facultad de Arquitectura y Bellas Artes, Pontificia Universidad Católica de Chile, 1990. SAN MARTín, Eduardo. “El Programa de Autoconstrucción de La Reina, Santiago de Chile". DANA (26): 69-79, 1988. SAN MARTÍN, Eduardo. La arquitectura de la periferia de Santiago: experiencias y propuestas. Santiago, Editorial Andrés Bello, 1992.

TURNER, John. Housing by people: Towards Autonomy in Building Environments.

Londres, Marion Boyars, 1976.

TURNER, John y FICHTER, Robert. Freedom to Build; Dweller Control of the Housing Process. Nueva York, Macmillan, 1972.

UNITED NATIONS HUMAN SETTLEMENT PROGRAMME. The Challenge of Slums [en línea]. Londres, Earthscan Publications, 2003 [fecha de consulta: 08 de enero de 2014]. Disponible en: <http://www. unhabitat.org/pmss/listltemDetails. aspx?publicationID=1156> UNITED NATIONS HUMAN SETTLEMENT PROGRAMME. State of the World's Cities Report 2012/2013: Prosperity of Cities [en línea]. Nueva York, Routledge, UNHABITAT, 2012 [fecha de consulta: 8 de enero de 2014]. Disponible en: <http://www. unhabitat.org/pmss/listltemDetails. aspx? publication ID =3387>

ZERÁN, Faride. Tiempos que muerden: biografía inconclusa de Fernando Castillo Velasco. Santiago, LOM, 1998. 
FRANCISCO QUINTANA | ARCHITECT, PONTIFICIA UNIVERSIDAD CATÓLICA DE CHILE, CHILE.

\section{OPERATION SITE AND}

\section{VILLA LA REINA [1965-1970]}

Between 1940 and 1960, the population

of Santiago doubled and produced a housing crisis within the capital. While President Eduardo Frei Montalva (19641970) set out to combat the housing shortage, the government would have neither the money nor the time sufficient to achieve this objective with traditional public policy. So they implemented a plan, nicknamed by its opponents as "Operation Chalk" in the mid-sixties politicians and planners decided not to build houses for those living in extreme poverty, but to give them ownership of a site outlined in chalk. Given the urgency and lack of funds, they opted to provide that which the people could not obtain themselves: urban design, access to services and basic infrastructure and connection to the city networks. Despite difficulties (and not without mistakes) over a period of five years "Operación Sitio" -Operation Site- (the official title of this policy) gave close to 71 thousand sites to more than 380 thousand people, extensive urbanized areas on the outskirts of Santiago during the second half of the ' 6 os.

Villa La Reina, a large neighborhood in the Eastern Santiago, is a self-built project developed during this period. Unlike "Operation Site," this complex was developed at a local scale, led by the architect Fernando Castillo Velasco, mayor of La Reina from 1964 to 1968 . The collaborative work between the mayor's office, the local community and the Universidad Católica, was one of the key aspects of this project. The organization of its residents was not only relevant to the construction of the project, but also to the design process and the creation of an integral neighborhood with adequate public space, provided with educational facilities, close to new sources of employment and connected to the structure of the city.

While "Operation Site" and the self-construction seemed like viable strategies for reducing costs and accelerating the supply process, they were severely criticized. In terms of location, it was foreseen at the beginning of the "70s that "Operation Site" would result in large-scale social segregation. In terms of self-construction, Salvador Allende's government would opt for eliminating this type of program, stating that it was an inefficient process and that it discriminated against a population with scarce resources.

\section{FIRST HALF OF THE 20TH CENTURY:} MIGRATIONS AND HOUSING DEFICITS Large migrations to the cities characterized the first half of the $2 \mathrm{O}^{\text {th }}$ century in Chile. If in 1907 , only $38 \%$ of people lived in cities (1.2 million), by 1970 this percentage had increased to $71.6 \%$ (6.4 million) (Geisse, 1983). This is largely due to migrations from rural areas and the bankrupt saltpeter mines ${ }^{1}$. Santiago, the financial and administrative capital of the country, attracted the majority of these migrants. The result was a huge shortage of housing in the mid-sixties.

The city of Santiago, since the early $2 \mathrm{O}^{\text {th }}$ century, was gradually transformed into the largest industrial center of Chile. In 1916, $45 \%$ of public investment in infrastructure was concentrated in the capital, a city with just $18 \%$ of the country's population (Geisse, 1983) (fig. 1). The following generation of employment in Santiago was an attractive destination for relocation. In 1907, the city had a population of 300 thousand inhabitants. In 1940, this figure had increased to 950 thousand and by 1960 , this number had doubled to 1.9 million people: $58 \%$ of the urban population in the country concentrated in Santiago producing a housing crisis. Those with scarce resources arrived at the capital seeking employment and education opportunities and had no alternative but to live in tenements or in so-called "poblaciones callampa", shanty-towns.

The tenements were precarious dwellings by developers who rented to low-income people. They consisted of two rows of rooms sharing a small alley. The lack of ventilation, clean water and plumbing was common in these developments and over-crowding was part of daily life (CORVI, 1962) (fig. 2). Meanwhile, the "callampas" were informal and illegal settlements on public or private land, generally in deteriorated spaces in the city. In 1952, close to 35 thousand dwellings made up different shantytowns in the country's cities, occupying vacant lots mostly in Santiago (CORVI, 1962). The main objective for these settlements was to be able to live closer to employment and education opportunities offered in cities (fig. 3).

In 1953 the Corporación de la Vivienda (corvi) was created to provide housing solutions throughout the country; at that time, the housing deficit in Chile would reach more than 145 thousand units (corvi, 1963). The institution would address the needs of both the middle class and the settlements with scarce resources, and the projects promoted by this entity would include housing projects, like Salar del Carmen in 1960 -designed by Mario Pérez de Arce Lavín and Jaime Besa (fig. 4)- to tower complexes and blocks, like the Unidad Vecinal Portales, designed in 1958 by the office of Bresciani, Valdés, Castillo and Huidobro (fig. 5).

By the mid-fifties, the CORVI began to apply strategies that would be the precursors to "Operation Site". The self-construction and eradication programs consisted in the elimination of the "callampa" settlements, motivated by the unhealthy environment in which the inhabitants were living. CORVI acquired the land needed to eradicate the inhabitants and give them the title to a new site. The lot contained a small bathroom and kitchen installation connected to basic services.
The settlers themselves would build the most of their definitive dwellings (CORVI, 1963) (fig. 6).

Self-construction emerged informally in several Latin American countries during the ' 50 s and ' 60 s. These settlements were called "barriadas" on Perú, "villas miseria" in Argentina, "favelas" in Brazil, and "poblaciones callampa" in Chile $^{2}$. The architect, John Turner, studied the self-construction process on site and promoted it internationally as a feasible solution to the housing problem of those in extreme poverty. According to his observations in Lima, the strategy behind self-construction was more appropriate than the solutions provided by the government and private enterprise. First, it was a more economical solution than those by traditional means. The settlers supplied the labor for building the homes that responded more precisely to their needs and not just a type house. Finally, during the process the necessary social networks were developed for the future development of the complexes. Turner argued that all kinds of public institutions should support these constructions by means of technical support, urbanization, sites and building materials (Turner, 1976; Turner and Fichter, 1972).

THE RIGHT TO HOUSING IN THE EDUARDO FREI MONTALVA ADMINISTRATION.

During the Eduardo Frei Montalva administration, housing was defined as "a primary need that every family has a right to... whatever their socio-economic level may be" (Haramoto, 1980, p. 29). Following this plan, the State would economically assist the low-income families that would be unable to acquire housing alone. In the case of people living in extreme poverty, the state would not only finance the whole dwelling, but also would undertake part of the self-construction process. The idea seeks to integrate the population with the city networks, preventing that they continue living in informal settlements with inefficient spatial distribution, unhealthy conditions given their precariousness and the absence of utilities such as clean water and plumping. This would be promoted as "Operation Site".

In 1965 the government created the Chilean Housing and Urbanism Ministry (MINVU), responsible for, among other things, developing housing and urban plans. The programs elaborated at this time were not aimed at reducing the housing crisis to a housing deficit problem, but had the objective of creating integrated building complexes, thus building the facilities necessary for social development. Schools, clinics, sport fields, among other infrastructure, were understood as part of the housing problem (Palma y Sanfuentes, 1979). Meanwhile, the CORVI became an institution within this ministry and its responsibilities were limited to public housing.
Between 1964 and 1970 the housing policies aimed at building sixty thousand units a year, that is, 360 thousand definitive dwellings (Haramoto, 1980). By the mid-seventies, the average Chilean family was 5.4 people, that is, if you wanted to end the housing crisis, they need to build a city for close to two million people in six years. Of these dwellings, 213 thousand corresponded to low-income areas. The situation worsened after the strong storms of 1965 that had devastating effects in the central valleys: the social pressure for housing increased steeply and the untraditional yet necessary measures were taken to answer the deficit in a short amount of time and with a limited budget.

\section{FROM OPERATION SITE}

\section{TO OPERATION CHALK}

"Operation Site" began in 1965 as an emergency plan in response to the damage caused by devastating storms. Two years later, with the Low-income Savings Plan, it would become a formal channel to provide housing to low-income citizens. The program essentially consisted of providing loans to buy single-family plots with utilities and connected to the city where the inhabitants would build their own homes. Following the tenets of the MINVU, these complexes would be planned with proper education, recreation and health facilities. Between 1965 and 1970 around 71 thousand sites were delivered to benefit more than 380 thousand people (Garcés, 2002, p. 308). However, the quality of these plots was inconsistent so that residents and opponents of the policy began to call it "Operation Chalk" as in some cases these sites consisted in no more than a chalk outline.

By 1962 , the CORVI reports indicated that $76 \%$ of the housing construction investment was spent on building, while the site cost $5 \%$ and urban development cost $19 \%$ of the total investment (CORVI, 1962; CORVI, 1963). Thus, the self-construction process would reduce the initial investment to provide a solution to low-income settlers that had no capacity for savings to access a definitive dwelling by traditional means. "Operation Site," therefore, would accelerate the distribution process of the sites, bringing housing access to the masses through incremental construction by the settlers.

The Low-Income Savings Plan consisted in five options ${ }^{3}$, the first and most basic corresponding to "Operation Site". The first stage consisted in a 160-m2 lot, which in the best case could have a mediagua ${ }^{4}$ located at the back. Urban development was reduced to gravel roads and water lines on pylons and electrical lines providing street lighting and energy. The facilities were defined as a school, community center, local shops and open space. In the second stage the development would be completed with the installation of plumbing, water and electricity. The construction of the 
dwelling would be the owner's responsibility (Labadía, 1970).

The CORVI fulfilled the function of providing the necessary sites to develop the operations. The subdivisions built between 1966 and 1970 were homogeneously distributed in the peripheries of the capital, the majority along the Américo Vespucio loop, and inter-district freeway that enabled city expansion (Palmer y Vergara, 1990) (fig. 7). In these subdivisions urban design was reduced to the minimum to achieve maximum economic efficiency. The objective was to distribute the greatest quantity of lots and public spaces in the smallest area possible, maximizing the number of sites that would be serviced by the streets they faced. Increased efficiency is achieved with a rectangular lot with the smallest possible front to connect the most houses possible to plumbing and electrical lines, reducing the construction costs of linear meters of urbanization (fig. 8-13).

It must be noted that "Operation Site" is not considered as just housing construc tion, but included, at least in plan, the construction of schools, health centers, sporting areas, public space and commercial areas among other infrastructure. A prefabrication system would be tested for the construction of schools, while the Ministry of Education would supply the teachers (Garcés, 2002). The biggest problem was that the promised facilities were not built in all cases (Hidalgo, 2005); it would not be until the ' 80 s and onwards that different administrations would slowly improve the urban conditions in which these subdivisions were found.

"Operation Site" received severe criticism, not only because self-construction was considered discriminatory to those with scarce resources, but also for its location. Authors such as Manuel Castells (1971) argued, at the beginning of the ' 70 s, that this was the beginning of a massive social segregation. This fact increased exponentially due to the public policies adopted in the following decades and the continued expansions of Operation Site of socially homogenous tracts devoid (in many cases still today) of facilities, infrastructure and the minimum services for human development: education, health, employment, commerce, green spaces, etc.

\section{A CASE EXAMPLE}

Villa La Reina ${ }^{6}$ is a neighborhood of 1,592 houses built entirely by their owners: from the houses to the green areas, schools, churches and public spaces, including streets and plumbing and water lines. It is the largest of the assisted self-construction project in the ' 70 , not only in Chile but also in all the developing countries. The collaborative work between the mayor, the community and the Universidad Católica, differentiates the Villa La Reina from the other complexes built by "Operation Site" or later programs such as "Sites and Services"? that, developed by national governments, distanced themselves from the local problems of the communities. The participation of the architect, Fernando Castillo Velasco, in the collaborative work was particularly relevant, who being the mayor of $\mathrm{La}$ Reina Municipality, professor and, later, President of the Universidad Católica, wa able to summon political and academic institutions with the needs and expectations of a community organized to demand their right to housing.

\section{THE CREATION OF THE LA REINA DISTRICT: SOCIAL TENSION AND SEGREGATION}

During the ' 50 , the Nuñoa district grew rapidly with the arrival of diverse social classes, the majority coming from middle and upper class areas. Its proximity to the central districts of Santiago, where the greater sources of employment and commerce stood, attracted people to build homes in this area. On the other hand, and quickly, industrial areas emerged, providing jobs and attracting low-income settlers who began to illegally occupy public and private sites.

Over time, the social tension among the various residents of Nuñoa began to increase. Middle and upper class groups settled down in the Andean foothills and began to demand different treatment from the mayor. Their demands escalated to the presidency of Alessandri, who in 1963 decided to definitively separate the area from the rest of Nuñoa to found the Municipality of La Reina. This was not the only act of segregation. Oscar Castro, the first mayor, ordered the sites occupied by illegal squatters to be fenced, forcing them to relocate and avoiding new illegal occupations in the district (San Martín, 1988).

In 1964, President Eduardo Frei Montalva appointed Fernando Castillo Velasco, also a member of the Christian Democratic party, mayor of La Reina. His first actions as mayor had the objective on one hand to eliminate the segregation imposed in previous years, and one the other to promote social development in the district. Thus the Castro's decree was repealed and the Municipality began to develop the District Development Plan together with the Universidad Católica ${ }^{8}$. During his administration Fernando Castillo established the basis for an inclusive, integrated development for all socio-economic levels, incorporating industrial, institutional and cultural activities in the area of the city that was originally formed as a bedroom district. In his own words, "one day I came across 1,600 families that had no home and lived on the banks of the San Carlos Canal on vacant sites. I called to them and told them that they are members of the district and have the same rights as any other; they were people in extreme poverty, and I told them that I was committed to doing everything possible to make them the owners" (Cociña, Quintana, Valenzuela, 2009, p. 127). Thus, the settlers illegally occupying the degraded landscapes of the district were able to obtain a home. The incorporation of low-income housing within the development plan was fundamental to the incorporation of additional land-use in the area (Castillo, 2013).
The construction of Villa La Reina was an opportunity for those in extreme poverty to continue to live in the district, avoiding the relocation to places where they would lose the social and employment networks that had been created over the years. Thus, the first objective of the mayor was to find an affordable site for the settlers. Moreover, given the scarcity of resources, the self-construction through the organization of the local community appeared to be a feasible solution for reducing investment costs.

\section{LOCATION: CONNECTION}

\section{TO CITY RESOURCES}

The area selected for implementing part of the District Development Plan and the subsequent construction of the Villa was the estate of La Reina found in the center of the district. The site, a practically inactive agricultural area, contained 220 hectares belonging to the Social Security Service. Fernando Castillo, after meeting with different institutions and politicians, among them the then senator Salvador Allende, acquired the sites at a low price (Castillo Velasco, 2008, p. 25; Cociña, Quintana, Valenzuela, 2009, p. 127). The deal specified that $20 \%$ of the total area must be designated for the economically unstable population (Alvarado, 1967).

The District Development Plan established the construction of an industrial park made up of close to 100 low-impact industries and small enterprises, compatible with the housing development (Eliash, 1990). In this way, Villa La Reina would be located adjacent to a source of employment that would give work to the first generation of residents. The access to this new source of employment would greatly reduce one of the largest urban inequalities suffered in traditional social housing projects both in Chile and the world.

While La Reina is a peripheral district in Santiago, the Villa was located along Av. Larrain ${ }^{9}$, and inter-district artery that connects the neighborhood with the Américo Vespucio loop and with the more central districts in the capital. With the passage of time and with proper planning, this area was transformed into a sub-center annexing educational, cultural, recreational, and health facilities. Adding to this programmatic variety, the district has maintained through time its socio-economic diversity unlike the vast, isolated and socially homogenous tracts resulting from the abuse of "Operation Site" in areas such as Pudahuel and on the sites that today make up the district of Cerro Navia.

\section{URBAN DESIGN: THE BASIS FOR THE INCREMENTAL CONSTRUCTION OF A NEIGHBORHOOD}

Participatory design involving professors and students of the Universidad Católica School of Architecture and the future residents of the Villa La Reina was able to improve an urban design that pursued the same objectives of economic efficiency as those developed by "Operation Site" (fig. 14). In this case it was an urban design that was not only dedicated to the efficient distribution of lots but also to the appro-

priate distribution and design of streets, pedestrian paths, boulevards, plazas and the designation of commercial zones, schools and markets, along with other facilities. One of the objectives of the academic studio conducted by Fernando Castillo Velasco and Mario Pérez de Arce Lavin with the residents was to avoid the problems presented in other social projects of the time but to turn the Villa into “just another neighborhood," integrated into the city (San Martín, 1992; Castillo, 2013). The architect Renato Parada continued the result of these studios. An important figure in the administrative, design and construction processes was Eduardo San Martín, Project Director of the Municipality during this time.

Villa La Reina would not be made up of just houses, but would have other facilities such as a school built within the first year (fig. 15) and commercial areas. These community spaces would be located along Av. Larrain, the district's main street, with entrances into the Villa, with the goal of creating a better relationship between the neighborhood and the immediate urban context of the city (San Martín, 1988). Other facilities like the market and church, also built by the residents, were located within the Villa together with its main street.

In terms of public space, architects and residents decided to avoid large square plazas that generally deteriorated into delinquent areas. On the contrary, small plazas were distributed with the houses and pedestrian paths toward the interior of the Villa. The main green space would be a wide, tree-lined sidewalk located along the neighborhood's main street, concentrating urban transport and the majority of urbanization costs (San Martín, 1988). This greenbelt distances the houses from the street while its width allows for it to be maintained by the neighbors themselves. These public spaces are relevant given that they are natural extensions of the dwellings and facilitate even more interaction among residents. The social cohesion and urban identity are relevant in the care and progress of the neighborhood.

The definition of the housing and its distribution are the results of academic studios and the work of the residents and architects of the municipality. The community rejected the first two designs because the houses were too small (Alvarado, 1967). Continuous blocks like row houses, similar to those of Operation Site, were not accepted either given that the residents aspired to detached houses. 
At the beginning of 1965 the residents began to organize the "Federación de Pobladores", made up of the 1,600 families in 16 committees who would be in charge of the development and construction of their own homes. Collectively, they laid down statutes, establishing, among other things, that one or two people per family would work only on Saturday, Sunday and holidays without knowing which dwelling would be theirs until they were all finished. The houses would be distributed by means of a family ranking according to their involvement in the construction and administration of the work. Technical assistance was provided at the beginning by the Municipality and later would be joined the technical institute, INACAP; civil engineering students from the Universidad Católica collaborated by overseeing construction. In May of 1966, the residents finished the model house and in August of the same year began construction on the rest of the complex (Alvarado, 1967).

The self-construction process was planned as an opportunity for the residents two learn new job schools as well as for creating companies by the neighbors themselves. INACAP conducted weekly training courses for site managers and assistants. Meanwhile, the municipal authorities encouraged the community to form their own industries and thus began the installation of factories for windows, doors, plasterboard, plumbing, prefabricated flooring and trusses. With the earth on-site they manufactured their own bricks (fig. 18-22). As the companies emerged to build the houses and develop the neighborhood, they produced a surplus of materials to be sold to other housing developments in Santiago and became involved in the construction of other projects in the district such as banks and supermarkets and even the Parque Industrial neighboring the Villa (Alvarado, 1967; Haramoto, 1980; San Martín, 1988; San Martín, 1992).

Self- construction was established in Villa La Reina as a way to create a sense of belonging and commitment to the project while also instilling the people of their own capabilities (Márquez, 2006). What began as a technical strategy for minimizing investment costs became a channel for social integration for low-income families into both the national economy and political spheres for the development of their social and built environment, overcoming marginalization (San Martín, 1992; Castillo Velasco, 20o8; Zerán, 1998).

\section{THE RIGHT TO A DECENT HOME. THE SAL-} VADOR ALLENDE ADMINISTRATION

\section{Salvador Allende, like Eduardo Frei}

Montalva, established during his administration (1970-1973) that housing would be an inalienable right for the people, but unlike the previous administration, he added that "it is the obligation of the State to provide housing for the people and that it should not be a source of profit" (Haramoto, 1980, p. 33). The new position of the government sought to deliver housing and eliminated this form of self-construction and incremental housing. "Operation Site" was criticized during this period for the reduced quality of the solution delivered and the lack of involvement by those affected in the design process (Hidalgo, 2005). Self-construction, on the other hand, was understood an unnecessary delay in the execution of the work and an inefficient use of economic resources (Palma y Sanfuentes, 1979).

The new policies adopted by the socialist government weakened state support for the Villa La Reina while social dynamics were fragmented and politicized (Márquez, 2006). However, the self-construction of the Villa did not stop thanks to the state of advancement and the social organizations that did not want to modify their work structure (San Martín, 1988).

Informal settlements continued to appear in the capital despite the efforts of Salvador Allende's government to reduce the housing crisis. These occupations had been on the rise since the Frei administration, increasing from 4 sites in 1968 to 104 in 1970 with an approximate population of 350 thousand people. By 1973 , the quantity of people living in camps reached 5oo thousand (De Ramón, 1990).

\section{HOUSING AND CONSUMERISM. THE DICTATORSHIP OF AUGUSTO PINOCHET (1973-1990)}

With the arrival of Augusto Pinochet to power, housing ceased to be an inalienable right and became a "right that is acquired by effort and savings" (Haramoto, 1988, p. 35). In the " 70 os and ' 8 os, as the neo-liberal economic model was adopted, the state began to take on a subsidiary role with respect to social housing and private enterprise became responsible for finding the land for new construction. Under this logic the land market is liberalized in 1979, arguing that the increase in available land will lower prices and facilitate the construction of more social housing, which did not occur (Hidalgo, 2005).

The social segregation that had germinated with Operation Site was greatly increased after the liberalization of the land market. The speculation that brought this measure considerably increased land value toward the center of the city, particularly affecting the informal camps located in these areas. A policy for eradicating the camps was implemented to move these communities away from the city center and towards the peripheries, concentrating poverty in districts that lacked the necessary health and education facilities and the necessary employment for development. Between 1979 and 1985 more than 170 thousand people were relocated, expanding Santiago at a rate of 3,ooo hectares annually (Hidalgo, 2004). During this time, the leaders of Villa La Reina were persecuted, the neighboring industries closed and the organizations dissolved (San Martín, 1988; Márquez, 2006). The residents finished individually the few houses that were still to be built in 1973 , located toward the east of the complex, having some support from the administration (Castillo, 2013, p. 98).

\section{HIDDEN INFORMALITY}

The migration to the capital in search of work and educational opportunities continued to increase during the " 70 s and ' 80 s. The formation of new informa settlements was prohibited and the cities were controlled severely by the military. The people, facing the social housing deficit and the impossibility of taking over vacant land, began a densification process within the small housing solutions provided in previous years by Operation Site and other policies. A single lot could receive two or three families on their property. A strong densification in low-income neighborhoods began. The social segregation described continued to grow, now from overcrowding, and the necessary infrastructure or connections with city resources were still unbuilt.

Villa La Reina was not exempt from this densification dynamic. Over time, lightweight expansions appeared in the back patios and later they became permanent; the original residents received the families of their children and other relatives (Castillo, 2013) (fig. 23). The socio-economic data shows that in Villa La Reina, by 2002 , close to $40 \%$ of the residents that continued living in the Villa had joined the middle class. According to the investigation by Francisca Márquez, between 2006 and 2009, social mobility and professionalism could be observed by the second generation, but they continued to live in the same district (fig. 24-29).

\section{URBANIZING WITH CHALK}

Facing the urgency and lack of resources, the strategy behind "Operation Site" and Villa La Reina sought to resolve the housing crisis by providing the basics for people that could not obtain them on their own. The population living in extreme poverty and illegally would be land owners, and without the fear of eviction, could improve their home over time along with the urban surroundings. If the site they were granted happened to be where they already lived, they could maintain their social networks and employment. Access to basic services such as clean water and plumbing would improve the quality of life of the residents. Urban design, as simple as it was, could maintain a certain order to further developments within the neighborhood and could avoid inefficiency, unsanitary conditions and insecurity typically found in spontaneously generated informal settlements (United Nations Human, 2003). It would also plan the construction of community facilities such as schools, sport fields, green spaces,

commercial zones and health centers that would promote social development. The land, within and connected to the city, would create the possibility of accessing job opportunities generated in urban centers. The self-construction process would provide new professional tools to the population while the collective work would encourage the creation of an urban identity of social networks and mutual aid.

Large urban migration continues to be a problem today, maybe not in Chile, but in the rest of the world. In the last decades, cities in Asia, Africa and Latin America grow by 800 thousand, 230 thousand and 150 thousand new inhabitants per week respectively (United Nations, 2012, p. 29). Strategies similar to "Operación Sitio" continue to be implemented in countries such as Pakistan and India to avoid the formation of informal settlements. We hope they repeat the successes and not the mistakes. ARO

Notes

1 In 1930, agriculture employed $37.5 \%$ of Chilean work force, but in 1970 -because of industrial farming- that ratio went down to $25 \%$. In the early '20s, saltpeter mines in the north of the country had attracted more than 65 thousand people; most of them moved back to the central valleys once the nitrate mining industry went bankrupt a few years later (Geisse, 1983).

2 In Rio de Janeiro, currently $20 \%$ of the population lives in "favelas" whilst informal urban areas in Caracas and Lima correspond to $60 \%$ and $70 \%$ respectively (Jáuregui, 2009).

3 These five options would be: semi-urbanized lots, urbanized lots, self-construction basic housing, single-storey $45 \mathrm{~m} 2$ units and apartments within 4-storey blocks (Labadía, 1970, p. 429). 4 In Chile, a "mediagua" is a basic dwelling unit intended to be used mostly for emergencies. It consists in a single slope roof wooden structure that encloses an area of $6 \times 3 \mathrm{~m}$. The interior space is barely finished.

5 The book El lote $9 x 18$ en la encrucijada habitacional de hoy, by Montserrat Palmer y Francisco Vergara, showcases a detailed research on the architecture of popular urban developments built in Santiago between 1959 and 1988

6 The most documented research on the history of Villa La Reina-from its foundation until the ' 2000 is available in the doctoral thesis research notes by María José Castillo Couve (2013). (See also Alvarado, 1967; Castillo Velasco, 2008; Eliash, 199o; Márquez, 2006; San Martín, 1988; San Martín, 1992; Zerán, 1998).

7 In the ' 7 os and ' 8 os the World Bank promoted and funded the "Sites and Services" program throughout number of developing countries in Latin America, Africa and Asia. The program, just like "Operation Site", provided urban lots to the poorest families of each country.

8 At the Universidad Católica School of Architecture, "the students Enrique Browne, Carlos Buchholtz, Sven Jacob and Nicolás Manase developed the plan as part of a undergraduate seminar led by professo Nicolás García and the professor and mayor of La Reina Fernando Castillo Velasco" (Castillo, 2013, p. 89).

9 Villa La Reina is located within the polygon defined by Larrain Ave. and Diputada Laura Rodríguez St. 